\title{
Fault diagnosis of downhole drilling incidents using adaptive observers and statistical change detection
}

Willersrud, Anders; Blanke, Mogens; Imsland, Lars; Pavlov, Alexey K.

Published in:

Journal of Process Control

Link to article, DOI:

10.1016/j.jprocont.2014.12.010

Publication date:

2015

Link back to DTU Orbit

Citation $(A P A)$ :

Willersrud, A., Blanke, M., Imsland, L., \& Pavlov, A. K. (2015). Fault diagnosis of downhole drilling incidents using adaptive observers and statistical change detection. Journal of Process Control, 30, 90-103.

https://doi.org/10.1016/j.jprocont.2014.12.010

\section{General rights}

Copyright and moral rights for the publications made accessible in the public portal are retained by the authors and/or other copyright owners and it is a condition of accessing publications that users recognise and abide by the legal requirements associated with these rights.

- Users may download and print one copy of any publication from the public portal for the purpose of private study or research.

- You may not further distribute the material or use it for any profit-making activity or commercial gain

- You may freely distribute the URL identifying the publication in the public portal 


\title{
Fault diagnosis of downhole drilling incidents using adaptive observers and statistical change detection
}

\author{
Anders Willersrud $^{\mathrm{a}, *}$, Mogens Blanke $^{\mathrm{b}, \mathrm{c}}$, Lars Imsland ${ }^{\mathrm{a}}$, Alexey Pavlov ${ }^{\mathrm{d}}$ \\ ${ }^{a}$ Department of Engineering Cybernetics, Norwegian University of Science and \\ Technology, N-7491 Trondheim, Norway, \{anders.willersrud,lars.imsland\} @itk.ntnu.no. \\ ${ }^{b}$ Automation and Control Group, Department of Electrical Engineering, Technical \\ University of Denmark, DK 2800 Kgs. Lyngby, Denmark, mb@elektro.dtu.dk. \\ ${ }^{c}$ AMOS Centre of Excellence, Department of Engineering Cybernetics, Norwegian \\ University of Science and Technology, N-7491 Trondheim, Norway. \\ ${ }^{d}$ Department of Advanced Wells and Enhanced Oil Recovery, Statoil Research, N-3905 \\ Porsgrunn, Norway, alepav@statoil.com.
}

\begin{abstract}
Downhole abnormal incidents during oil and gas drilling causes costly delays, any may also potentially lead to dangerous scenarios. Different incidents will cause changes to different parts of the physics of the process. Estimating the changes in physical parameters, and correlating these with changes expected from various defects, can be used to diagnose faults while in development. This paper shows how estimated friction parameters and flow rates can detect and isolate the type of incident, as well as isolating the position of a defect. Estimates are shown to be subjected to non-Gaussian, $t$-distributed noise, and a dedicated multivariate statistical change detection approach is used that detects and isolates faults by detecting simultaneous changes in estimated parameters and flow rates. The properties of the multivariate diagnosis method are analyzed, and it is shown how detection and false alarm probabilities are assessed and optimized using data-based learning to obtain thresholds for hypothesis testing. Data from a $1400 \mathrm{~m}$ horizontal flow loop is used to test the method, and successful diagnosis of the incidents drillstring washout (pipe leakage), lost circulation, gas influx, and drill bit plugging are demonstrated.
\end{abstract}

\footnotetext{
${ }^{*}$ Corresponding author.
} 
Keywords: Managed pressure drilling, fault diagnosis, statistical change detection, adaptive observer, multivariate $t$-distribution, generalized likelihood ratio test

\section{Introduction}

Drilling for oil and gas is a high-cost operation, especially for offshore wells. Here large drilling vessels are used, or the oil and gas platform is designed with drilling capabilities. An unwanted cost driver is non-productive time (NPT), which typically is between 20-25\% of drilling time [1]. One of the major contributors to non-productive time is unforeseen incidents happening with the equipment on the rig, or downhole in the well. Early detection and isolation of an incident is of great importance [1], since early detection and mitigation can reduce the impact of an incident. Downhole incidents may in particular be challenging to detect, and to distinguish one type of incident from another may be even more difficult. Detecting, and isolating the type and position of downhole incidents as soon as possible is the subject of this paper. A key issue is to avoid false alarms as these in themselves could cause unplanned stops in drilling operation while investigations are carried out to confirm an event.

A schematic of the possible downhole incidents in a drilling system is shown in Fig. 1. The main components of the system are the drillstring rotating the drill bit, with circulating drilling fluid pumped down inside the drillstring that transports crushed formation cuttings out of the annulus. The following incidents are of specific concern and are studied in this paper:

- An influx of formation fluid (gas, water, oil), also called a kick, is probably the most critical downhole incident. This is caused by a lower pressure in the well than in the formation. A gas kick will reduce the hydrostatic pressure, thus further worsening the situation, and possibly leading to a dangerous full blowout. Detection of kicks is one of the most studied detection scenarios in drilling, see $[2,3,4,5]$, and the importance of early detection is evident.

- Loss of drilling fluid to the formation is referred to as lost circulation. This is caused by either a very high permeability formation, or by a fractured formation [6]. If large amounts of fluid is lost to the formation there may be problems maintaining a full fluid column in the annulus, which again may lead to an influx. 
- A pack-off is a build-up of formation solids around the drillstring, partially or fully blocking the flow of drilling fluid. The result is typically stuck drillpipe and risk of formation fractures [7].

- Drillstring washout is a hole in the drillstring caused by wear, which may cause leakage to the annulus. Such a weakness can result a complete twist-off of the pipe, resulting in extra three to twelve days of drilling [8]. A washout is difficult to detect at an early stage because changes in pressure and flow are tiny when the leakage is small.

- With additives mixed in the drilling fluid, there is a possibility of plugging and washout of the drill bit. Status monitoring of the drill bit is important in order to reduce downtime, where service and replacement of the bit is better planned with increased monitoring.

Different models and different methods have been applied for detecting and isolating different kinds of incidents. Simple hydraulics models and observers were used by $[4,5]$, a high fidelity model was fitted to data in $[9,10]$, and [11] applied a knowledge-modeling method. Due to measurement noise, a statistical cumulative sum (CUSUM) algorithm was tested on flow measurements in [2], and in [12], skewness of the statistical distribution was used to detect poor hole cleaning. Estimation and diagnosis has been demonstrated in process context in [13] for a wastewater treatment plant where a bank of parallel linear observers were used for direct fault detection and isolation in a nonlinear plant, considering uncertainty but not stochastic elements. In our application, with a high sampling rate, the computational burden of this approach would be heavy when a high number of parallel observers were needed to adequately represent the nonlinearities of the system and different cases of parameter changes due to incidents listed above.

This paper employs a computationally simple mathematical model of the process in a nonlinear adaptive observer [14] to estimate friction parameters and fluid flow. The estimates were found to follow a $t$-distribution and a dedicated generalized likelihood ratio test (GLRT) was developed for this particular distribution in [15]. This paper makes use of the adaptive observer and the GLRT algorithm for the $t$-distribution from [15], to provide a multivariate test statistic in order to distinguish between the various types of downhole incidents that could happen. The purpose of this paper is to determine which of the possible incidents have happened, to where in the well the issue can be localized and which magnitude the incident has, hence 
which severity it has. The paper investigates the particular signatures of the different incidents in the test statistics and it develops rigorous methods to obtain both isolation and localization with desired probabilities of detection and false alarm. This result is achieved after a detailed analysis of properties of the vector comprising the test-statistic and simultaneous analysis of estimated flow and friction parameters in the downhole process. The contribution of this paper is to find a vector-based evaluation method for the test statistic such that all of the types of incidents listed above can be diagnosed with convincing diagnostic properties. The paper demonstrates the efficacy of the method on data from a medium-scale horizontal flow loop designed and tested by Statoil ASA, and compares the performance of the $t$-distribution and vector-based evaluation methodology with that of a standard Gaussian detection approach from [16] and shows the new method to be clearly superior.

The paper is organized as follows. Details about the test rig are first presented, and an overview of the fault diagnosis methodology is given. The hydraulic model is then detailed in Sec. 4, and changes to the different states and parameters in the model due to different incidents are discussed. Then, the adaptive observer is introduced, and a multivariate change detection algorithm is suggested. Tests with flow-loop induced faults data are finally presented, and the paper is completed with a discussion and conclusions.

\section{Flow loop test setup}

The experimental rig is a water-based horizontal flow loop of 1400 meters, designed to emulate and test different contingencies, including gas influx, lost circulation, bit plugging, and drillstring washout. The test setup is designed by Statoil ASA, and is located at the International Research Institute of Stavanger (IRIS), in Stavanger, Norway. The experimental test rig was designed to capture the main fluid dynamics in a real drilling rig using managed pressure drilling as closely as possible. In this configuration, the annulus is sealed off, and a choke is used to control the back-pressure. The schematics in Fig. 1 illustrates the process and faults that can be imitated in the test rig.

A conventional piston pump is used to circulate the drilling fluid, and circular steel pipes of $124 \mathrm{~mm}$ and $155 \mathrm{~mm}$ inner diameter are used, for the drillstring and annulus respectively, giving typical values of volume and bulk modulus. The back-pressure pump is omitted in the installation. Instrumen- 


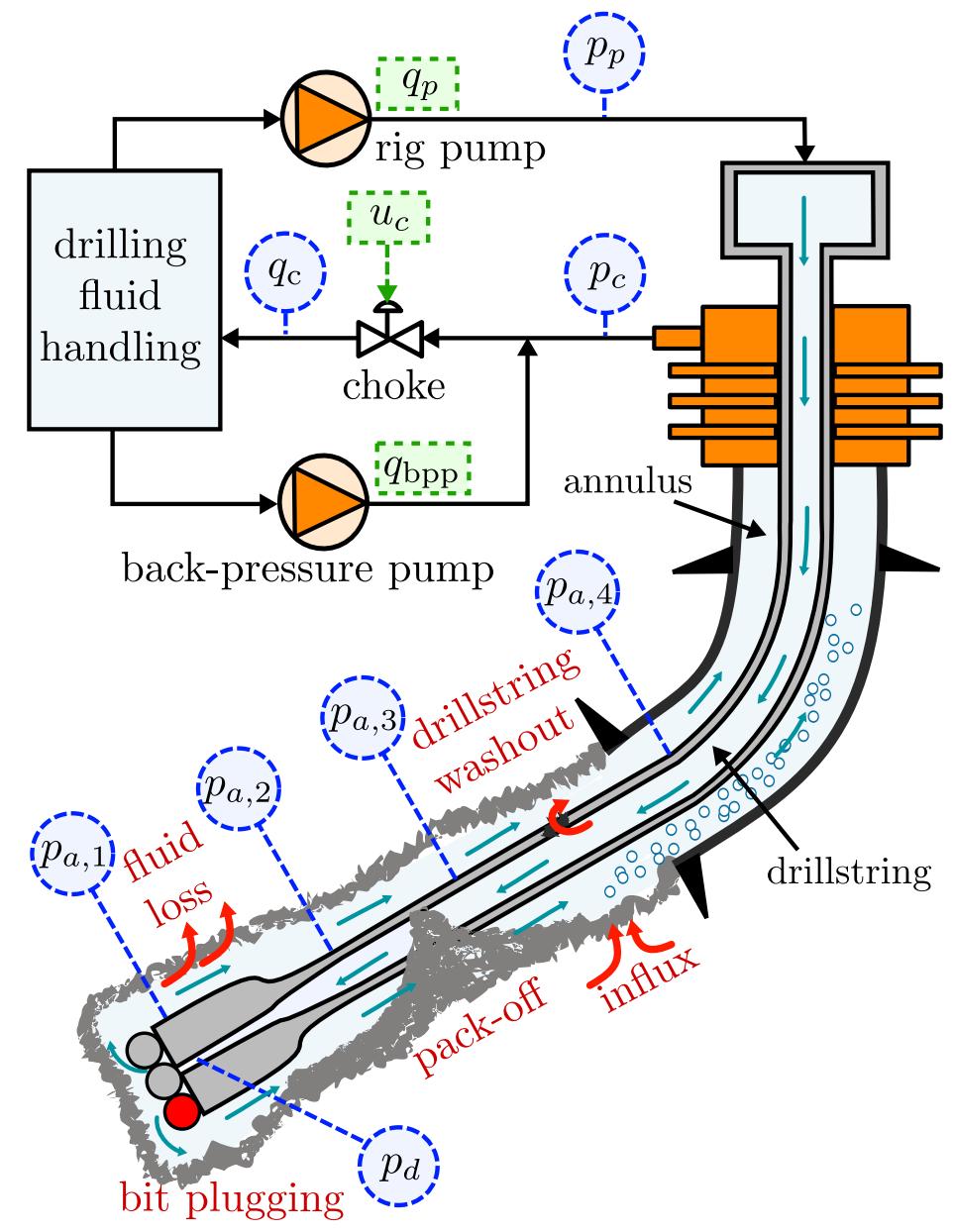

Figure 1: Drilling process with possible downhole incidents shown in red, including lost circulation, drillstring washout, formation fluid influx, bit plugging, and pack-off. Actuators shown in green, measurements in blue. 
tation is also typical for a real process. Pressure sensors downstream the pump (standpipe pressure), choke pressure, and pump flow are commonly available in a real rig. Downhole measurements along the drillstring and over the drill bit may be available with wired drill pipe technology [1, 17].
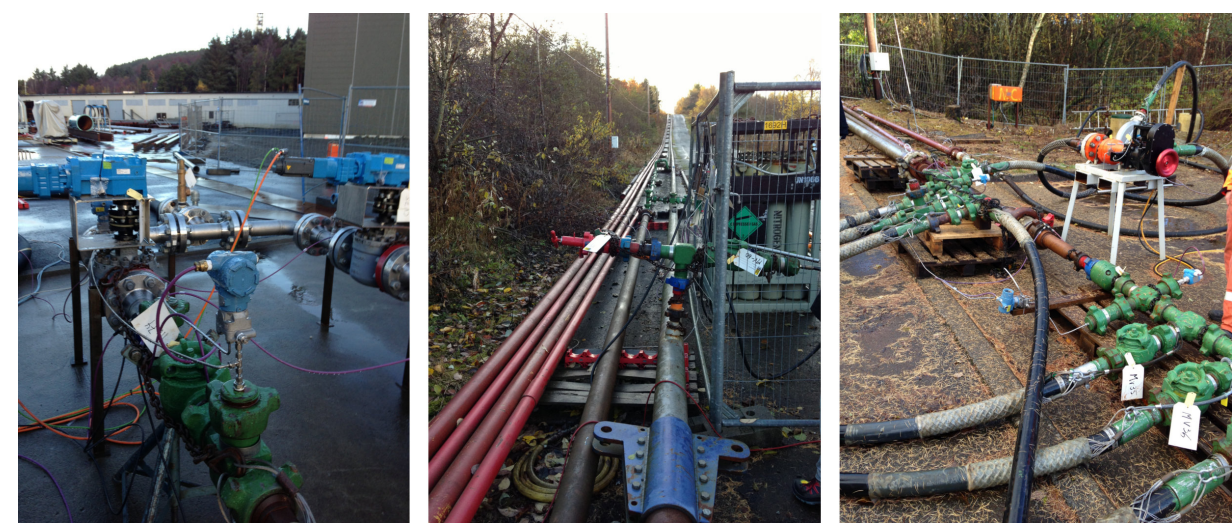

Figure 2: Experimental flow loop with choke manifold to the left, gas influx and washout emulation in the middle, and bit and loss emulation to the right.

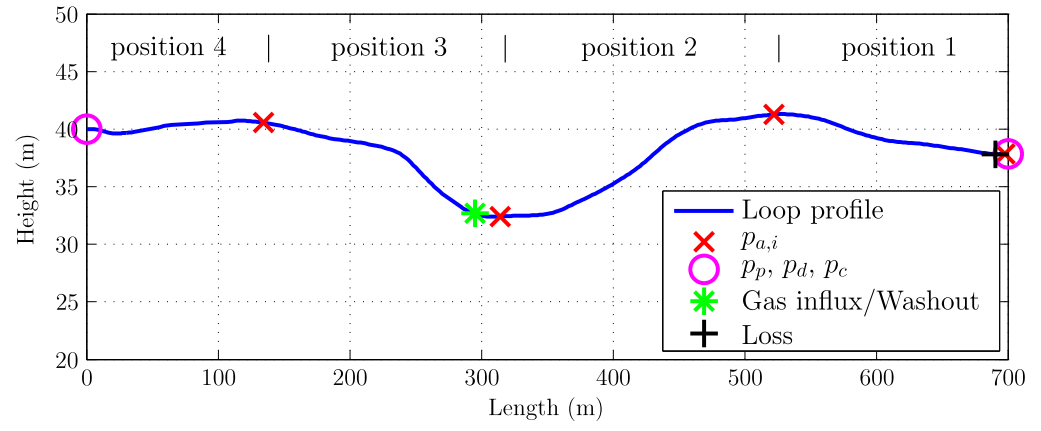

Figure 3: Flow loop profile showing location of incident emulation at different positions in between pressure sensors.

However, some aspects of a real drilling process will not be captured in the test rig, where one of the more noticeable differences is caused by the loop being close to horizontal. In an inclined well with up to thousands of meters height difference between top and bottom, the volume of a gas influx will increase as it is approaching the surface, due to decreasing pressure. The result is a decreasing pressure in the bottom of the well. This effect will be much less noticeable when the hydrostatic pressure differences in the 
well are small, which is the case with the test rig. However, note that this effect is only occurring during multi-phase flow, which is the case during an influx. Other aspects are the lack of crushed formation particles in the annulus, annular effects and effects due to drillstring rotation. Nevertheless, flow rates and volumes, as well as a high-pressure environment, are chosen to give flow dynamics similar to real drilling.

Key parts of the process that are emulated are: drill bit; choke manifold; gas influx; drillstring washout; lost circulation. These are illustrated in Fig. 2. Gas influx is emulated by nitrogen injection in the middle of the annulus. At the same location, a valve can be opened, rerouting the flow from the drillstring to the annulus to emulate a drillstring washout. In the end of the drillstring, three adjustable valves emulate the bit with the possibility of partial plugging. The loop profile is shown in Fig. 3, illustrating that the loop does have height differences and therefore a hydrostatic pressure that varies along the line. Location of the different incidents and pressure sensors are also shown in the figure.

\section{Fault diagnosis methodology}

This section presents the fault diagnosis method proposed for this problem. Fault diagnosis [18] consists of

- Fault detection: detect that an abnormal situation has occurred.

- Fault isolation: determine the type and location of the fault.

- Fault estimation: estimate fault magnitude.

Fault diagnosis methods can be divided into model-based methods using mathematical models of the system [19, 20, 21], and data-driven methods that only are dependent on measurements, which can be beneficial for large systems $[22,23,24]$. Data-driven methods for multivariate statistical fault diagnosis are presented and discussed in [22, 23, 25]. This paper presents a model-based multivariate statistical fault diagnosis method to detect and isolate the possible incidents. Tests are done on data from the test rig.

Generally, model-based fault diagnosis is based on detecting observable changes that occur due to faults in the system. These changes can appear in residuals, signals that are zero under normal conditions but differ from zero in the presence of faults, or in estimated parameters of the system. One 


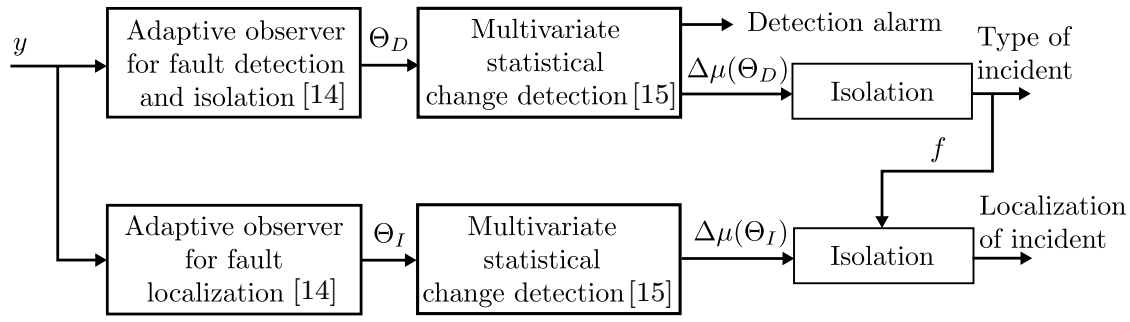

Figure 4: Fault detection and isolation based on parameter and state estimation, and statistical change detection.

approach to estimate parameters is to use adaptive observers, which estimate states and slowly varying unknown parameters. When the adaptive observer is designed to tolerate unknown input, both abrupt and incipient faults can be captured [26].

In this paper, fault diagnosis is done by detecting changes, compared to normal operating conditions, of estimated friction parameters and change in flow rates. These estimates will have a random component due to measurements noise propagating though the adaptive observer. Since the magnitude of the different incidents can vary from zero to an unknown magnitude, and the random component is significant, the generalized likelihood ratio test (GLRT) can be applied to detect the change [20, 27, 28]. The detection problem is to detect a change in signal $x$ from the null hypothesis $\mathcal{H}_{0}$ to the alternative hypothesis $\mathcal{H}_{1}$, and can be formalized as

$$
\begin{aligned}
& \mathcal{H}_{0}: \quad x \sim \mathcal{D}\left(\Pi_{0} ; \mathcal{H}_{0}\right), \\
& \mathcal{H}_{1}: \quad x \sim \mathcal{D}\left(\Pi_{1} ; \mathcal{H}_{1}\right),
\end{aligned}
$$

where $\mathcal{D}\left(\Pi_{i} ; \mathcal{H}_{i}\right)$ is the probability distribution of $x$ with statistical parameters $\Pi_{i}$ specified at $\mathcal{H}_{i}$.

The paper focuses on investigation of a methodology to isolate the type of fault that has occurred, to locate where in the well the fault is present and to estimate the magnitude of the incident, i.e., help to assess the severity of the incident. The fault diagnosis methodology is presented graphically in Fig. 4. As indicated in the figure, details about design of the adaptive observers are available in [14] and derivation of the GLRT detector for a $t$-distribution is available in [15].

Detecting that a fault is occurring, and determining the type of fault $f \in \mathcal{F}$, is based on estimated states and parameters in the friction parameter 
observer, using available measurements $y$. The location of the fault is found by the help of the flow estimation observer. Due to noise in the estimated signal, changes are detected using a multivariate statistical change detection algorithm. A univariate test on each estimated parameter would be a possibility, as was done in [16], but this paper shows it is possible to achieve much better detection properties using a multivariate method where all parameters are considered jointly [15]. An alarm is set if the test statistics exceeds a certain threshold. Isolation is done by determining the change direction of the estimated parameters and states, where different faults will give different directions. This approach is similar to [22], where isolation was based on vectors in a data-driven principal component analysis (PCA) framework.

\section{Simplified single-phase hydraulics model}

The flow loop was rigged for managed pressure drilling (MPD), and therefore is a model designed for MPD used. Referring to Fig. 1, the model can easily be changed to conventional drilling by removing the choke and backpressure pump.

The process model [29] is a simplified hydraulics single-phase model with two control volumes connected with a momentum balance at the drilling bit. This model has been verified for offshore MPD commissioning tests, and is suitable for control and detection purposes where unknown parameters can be estimated. Slowly varying effects due to temperature are not included in the model, but can be added to calibrate the physical parameters. The height difference between a real drilling rig and the test rig will only affect the hydrostatic pressure during normal operation with single-phase flow, which will not affect the dynamics noticeably. The model is represented by the ordinary differential equations

$$
\begin{aligned}
\frac{d p_{p}}{d t} & =\frac{\beta_{d}}{V_{d}}\left(q_{p}-q_{\mathrm{bit}}\right), \\
\frac{d p_{c}}{d t} & =\frac{\beta_{a}}{V_{a}}\left(q_{\mathrm{bit}}+q_{\mathrm{bpp}}-Q_{c}\left(\theta, p_{c}, u\right)\right), \\
\frac{d q_{b}}{d t} & =\frac{1}{M}\left(p_{p}-p_{c}-F(\theta, q)-\left(\rho_{a}-\rho_{d}\right) g h_{\mathrm{TVD}}\right),
\end{aligned}
$$

where $p_{p}$ is the pressure downstream the rig pump, $p_{c}$ pressure upstream the choke, $q_{p}$ the volumetric pump flow, $q_{b}$ the flow through the bit, and $q_{c}$ the flow through the choke. In each control volume $j \in\{d, a\}, d$ for drillstring 
and $a$ for annulus, $\beta_{j}$ is bulk modulus, $V_{j}$ is volume, $\rho_{j}$ is fluid density, and $L_{j}$ is the length. The vertical depth of the well is denoted $h_{\mathrm{TVD}}$, and $g$ is the acceleration of gravity. The integrated density per cross section $M$ is given by $M=M_{d}+M_{a}$ where $M_{j}=\int_{0}^{L_{j}} \rho_{j}(x) / A_{j}(x) d x$. The total friction $F(\theta, q)$ is dependent on the unknown parameter vector $\theta$ of slowly varying parameters which will be estimated. Friction is represented by

$$
F(\theta, q)=\theta_{d} f_{d}(q)+\theta_{b} f_{b}(q)+\theta_{a} f_{a}(q)
$$

where $f_{d}(q), f_{b}(q), f_{a}(q)$ are the flow characteristics in the drillstring, over the bit, and in the annulus, respectively, and $\theta_{d}, \theta_{b}, \theta_{a}$ are unknown parameters. These parameters are lumped parameters of well geometry, density and viscosity, where the two latter again are functions for pressure and temperature. For normal operation these parameters can be assumed constant. The choke is modeled by

$$
Q_{c}\left(\theta, p_{c}, u\right)=\theta_{c} q_{c}\left(p_{c}, u\right)=\theta_{c} \operatorname{sgn}\left(p_{c}-p_{c, 0}\right) g_{c}\left(u_{c}\right) \sqrt{\left|p_{c}-p_{c, 0}\right|},
$$

where $p_{c, 0}$ is the pressure downstream the choke, and $g_{c}\left(u_{c}\right)$ is the choke characteristics as a function of choke opening $u_{c} \in[0,100]$. Let $p_{d}$ and $p_{a, 1}$ be the pressure measurements upstream and downstream the bit, respectively. Then the relationship between friction and pressure in the drillstring, over the bit, and in the annulus is, respectively,

$$
\begin{aligned}
p_{d} & =p_{p}-\theta_{d} f_{d}(q)+G_{d}, \\
p_{a, 1} & =p_{d}-\theta_{b} f_{b}(q), \\
p_{a, 1} & =p_{c}+\theta_{a} f_{a}(q)+G_{a},
\end{aligned}
$$

where $G_{d}=\rho_{d} g h_{\mathrm{TVD}}$ and $G_{a}=\rho_{a} g h_{\mathrm{TVD}}$. In addition, if measurements $p_{a, i}$ are available throughout the annulus, the relationship between pressure and friction is

$$
p_{a, i}=p_{a, i+1}+\theta_{a, i} f_{a}(q)+G_{a, i}, \quad i \in\left\{1, \ldots, N_{a}\right\}
$$

where $\theta_{a, i}$ is the friction parameter for the annular segment between measurement $p_{a, i}$ at depth $h_{a, i}$ and $p_{a, i+1}$ at $h_{a, i+1}$, with $G_{a, i}=\rho_{a} g\left(h_{a, i}-h_{a, i+1}\right)$ and $\theta_{a}=\sum_{i=1}^{N_{a}} \theta_{a, i}$. The vector of unknown parameters is thus

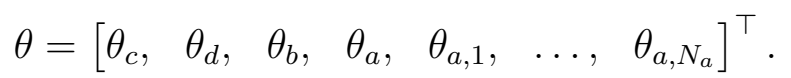


Data from a flow test with different flow rates is used to empirically determine the friction characteristics in the drillstring, over the bit, and in the annulus. In Fig. 5 friction losses for flow rates in the range of $270 \mathrm{~L} / \mathrm{min}$ to $1500 \mathrm{~L} / \mathrm{min}$ are plotted, showing a good fit to a quadratic relationship, i.e.,

$$
f_{d}(q)=q^{2}, \quad f_{a}(q)=q^{2}, \quad f_{b}(q)=q^{2},
$$

which is typical for turbulent flow, as well as for describing pressure drop over the bit [6].
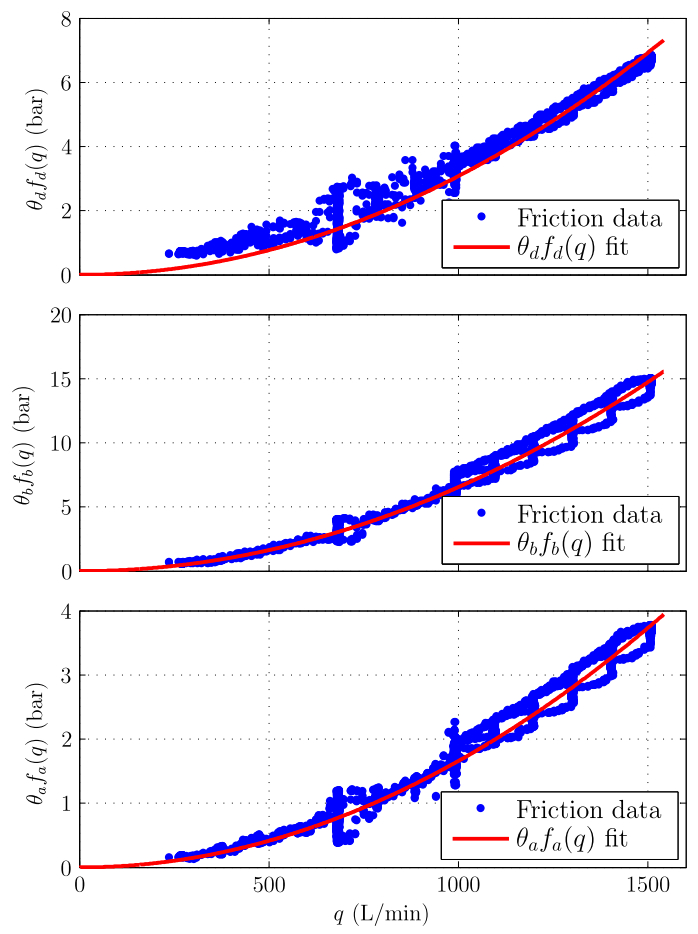

Figure 5: Friction characteristics in drillstring, drill bit, and annulus.

\section{Classification of incidents based on changes to variables}

The different downhole drilling contingencies studied in this paper are lost circulation, which is loss of fluid to the formation, influx of gas from the formation, drillstring washout causing leakage from the drillstring to the annulus somewhere in the well, drill bit plugging, and pack-off of formation cuttings around the drillstring, restricting flow. 
These different incidents will affect friction and flow throughout the well differently, and is used in the fault diagnosis method. Changes in mean of the estimated parameters $\hat{\theta}_{d}, \hat{\theta}_{b}$, and $\hat{\theta}_{a}$, as well as change in estimated flow in and out of the well,

$$
\Delta \hat{q}:=\hat{q}_{c}-\hat{q}_{p}
$$

are used to differentiate between the different incidents.

\subsection{Lost circulation}

Loss of fluid to the formation somewhere in the annulus will result in less flow downstream the point of loss. This will again reduce the friction in the segments with less flow, as well as the total annulus friction. Since the friction in the annulus is estimated by $\hat{\theta}_{a} f_{a}\left(\hat{q}_{\mathrm{bit}}\right)$, a reduction in annular flow will result in a reduction in the estimated friction parameter. This is due to the fact that annular flow is not estimated, but assumed equal to bit flow. These effects are illustrated in Fig. 6a, showing less flow in the annulus, causing less friction and a negative change of $\Delta \hat{q}$.

\subsection{Drillstring washout}

Drillstring washout is leakage from the drillstring to the annulus due to small holes in the drillstring. If washout happens, the lower parts of the drillstring and annulus will have reduced flow, which may result in decreased well pressure and hole cleaning capabilities. The effect on the friction parameters are shown in Fig. 6b, where friction in the lower parts of the well is reduced. At the section of the washout, the pressure in the end of the section will be constant due to unchanged flow, but the pressure in the beginning of the section will decrease due to reduced friction in the section. The net effect is an increase in pressure drop at the washout. The case of drillstring washout was thoroughly studied in [15].

\subsection{Gas influx}

An influx of formation gas into the well is an incident caused by pressure in the well being lower than the formation pressure. Pressure dynamics will change with gas in the well, requiring a more advanced hydraulic model than (2). As the gas percolates up the well its volume will increase due to a smaller hydrostatic head. This will give a smaller pressure drop and thus a smaller estimated friction if the change in density of mixed gas and liquid is not accounted for. However, due to reduced holdup for the liquid in the annulus, 


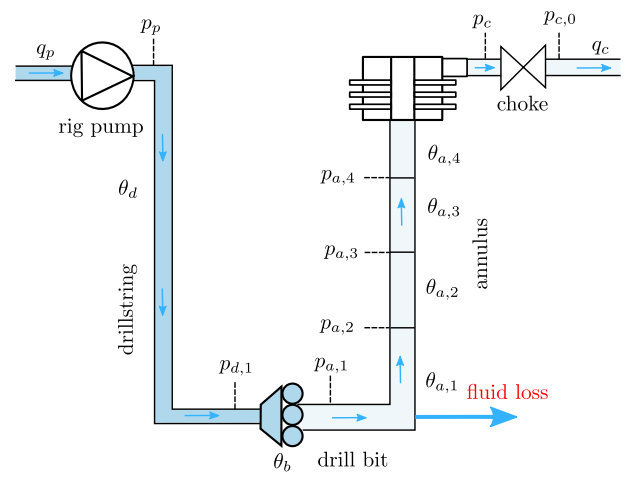

(a) Lost circulation.

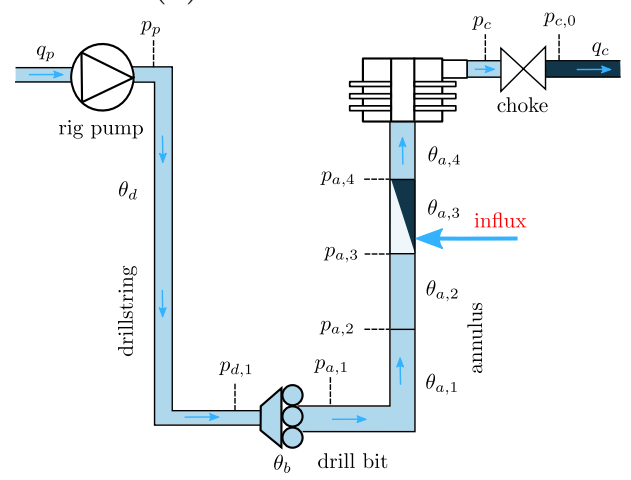

(c) Fluid influx.

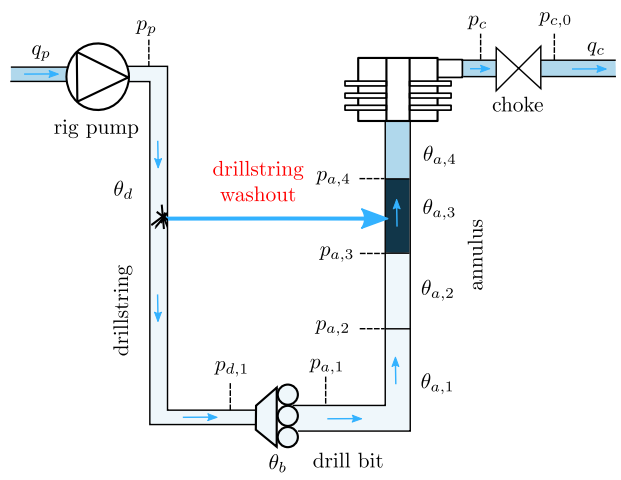

(b) Drillstring washout.

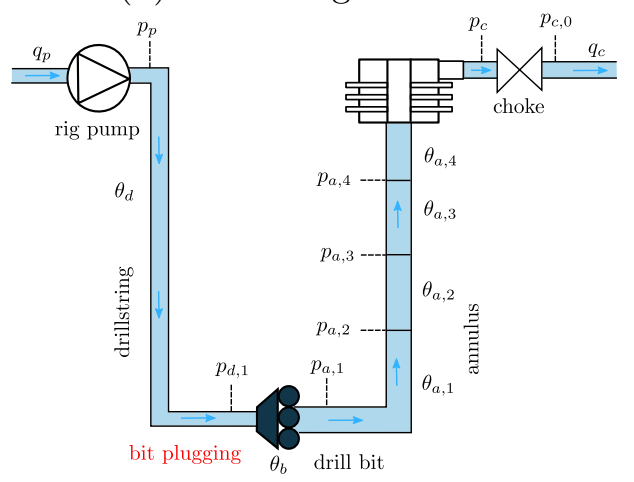

(d) Bit plugging.

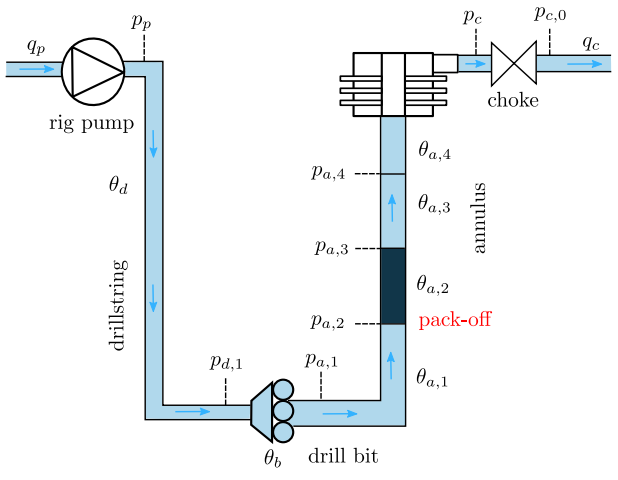

(e) Pack-off.

Figure 6: Changes to flow and parameters due to different incidents. Blue denotes normal flow, light blue is less flow and/or friction, dark blue is increased flow and/or friction. 
its velocity will increase, increasing wall friction. These two effects will either increase or decrease the pressure drop over the annulus, depending on the magnitude of circulating flow rate and well inclination. The multi-phase flow is often classified as either gravity dominated or friction dominated. For vertical multi-phase flow of gas and liquid, typically 90-99 \% of the pressure loss is caused by reduced hydrostatic head [30], i.e., gravity dominated flow.

An influx is thus associated with a decrease in $\Delta \hat{q}$, and change in $\hat{\theta}_{a}$, with positive change for friction dominated flow and negative for gravity dominated flow. In the particular case of the flow loop the inclination is quite small, hence is it assumed friction dominated flow in the annulus.

\subsection{Pack-off}

In addition to controlling pressure in the well, the drilling fluid is used to transport crushed formation particles (cuttings) or parts of the wellbore out of the well. If the drilling fluid fails to transport this mass, the wellbore can be (partially) plugged around the drillstring, called a pack-off. This will be observed in the friction parameters as an increase in $\hat{\theta}_{a}$, while the rest of the friction parameters and flow are unchanged. Pack-off is not emulated in the flow loop, but included here to demonstrate that other incidents are not incorrectly isolated as a pack-off.

\subsection{Bit plugging}

The drill bit has several nozzles which may be plugged during drilling. Small particles from the cuttings may restrict the flow through one or several of the nozzles, which will be seen as an increased pressure drop over the bit, and thus an increase in the pump pressure. Since the formation is not exposed to this pressure increase, the incident is not as severe as a packoff [9]. If pressure sensors are available on both sides of the bit, changes to the pressure drop can be used to indicate a plugging. However, changes to the corresponding friction parameter may be a result of other incidents happening. A salient feature of the method proposed here is that changes to the whole drilling process are considered simultaneously.

\subsection{Overview of changes due to incidents}

The effects on friction parameters and flow described in the previous subsections are summarized in Tab. 1, showing no overlap in the signatures that different faults have in the estimated parameter vector. A vector-based method can thus be applied to isolate the type of incident, using change 
directions based on this table. Depending on whether the pressure drop during a gas influx is gravity dominant or friction dominant, $\hat{\theta}_{a}$ will either decrease or increase during the influx. It is assumed that the pressure drop is friction dominated since flow loop is close to horizontal, giving a positive change for $\hat{\theta}_{a}$ in Tab. 1 .

Table 1: Change of estimates in different cases of faults. Legend: increasing $(+)$; decreasing $(-)$; unchanged $(0)$.

\begin{tabular}{lrccc}
\hline & $\hat{\theta}_{d}$ & $\hat{\theta}_{b}$ & $\hat{\theta}_{a}$ & $\Delta \hat{q}$ \\
\hline Lost circulation & 0 & 0 & - & - \\
Drillstring washout & - & - & - & 0 \\
Gas influx & 0 & 0 & + & + \\
Bit plugging & 0 & + & 0 & 0 \\
Pack-off & 0 & 0 & + & 0 \\
\hline
\end{tabular}

To isolate the position of the different incidents, changes to friction parameters $\hat{\theta}_{a, 1}, \ldots, \hat{\theta}_{a, N_{a}}$ are used. The position of the incident will affect the parameters differently, hence making isolation possible.

The estimated parameter vector $\Theta_{D}$ is used for detection and isolation of incident type, and $\Theta_{I}$ for isolation of position. The vectors are

$$
\Theta_{D}:=\left[\begin{array}{c}
\hat{\theta}_{d} \\
\hat{\theta}_{b} \\
\hat{\theta}_{a} \\
\Delta \hat{q}
\end{array}\right], \quad \Theta_{I}:=\left[\begin{array}{c}
\hat{\theta}_{a, 1} \\
\hat{\theta}_{a, 2} \\
\hat{\theta}_{a, 3} \\
\hat{\theta}_{a, 4}
\end{array}\right] .
$$

Two separate vectors are used since in general, it may not be possible or desirable to estimate $\Theta_{D}$ and $\Theta_{I}$ in the same observer. Furthermore, the magnitude of change due to an incident may differ between the two vectors. In this specific case, $\Theta_{I}$ represents only a part of the process and will give smaller changes during incidents compared to $\Theta_{D}$. Scaling would then be a challenge, as well as deteriorating detection and isolation properties, since a trade-off between false alarm and detection rate between $\Theta_{D}$ and $\Theta_{I}$ would have to be considered. 


\section{Parameter estimation using adaptive observers}

Two different observers are designed to estimate parameters in the drillstring process. These were illustrated in Fig. 4. One observer is used to obtain parameter estimated of type $\left(\Theta_{D}\right)$, which are used to detect and isolate the type of fault that has occurred; another is used to obtain estimate parameters, $\Theta_{I}$, which are used for fault localization. For the drilling case with distributed pressure sensors in the annulus, the difference between the observers will be that the detection and isolation observer estimates $\hat{\theta}_{a}$ for the whole annulus, whereas $\hat{\theta}_{a, i}, i \in\left\{1, \ldots, N_{a}\right\}$ is estimated in the localization observer. When using (2) as system model it is possible to estimate all these parameters simultaneously, and hence for simplicity of presentation they are presented in one observer in the current section.

The model (2) has nonlinearities in the choke equation (2e) and the friction $(2 \mathrm{~d})$. In order to estimate states and parameters, a nonlinear adaptive observer is applied. The observer was derived in [14], and successfully applied on the washout case in [15]. The model (2) can be written on the nonlinear adaptive observer form,

$$
\begin{aligned}
& \dot{x}=\alpha(x, u)+\beta(x, u) \theta, \\
& z=\eta(x, z)+\lambda(x, u) \theta,
\end{aligned}
$$

where $x(t) \in \mathbb{R}^{N_{x}}$ are the states, $z(t) \in \mathbb{R}^{N_{z}}$ are the additional measurements, $u(t) \in \mathbb{R}^{N_{u}}$ are the inputs, $\theta \in \mathbb{R}^{N_{\theta}}$ are unknown parameters, and $\alpha(x, u) \in$ $\mathbb{R}^{N_{x}}, \beta(x, u) \in \mathbb{R}^{N_{x} \times N_{\theta}}, \eta(x, z) \in \mathbb{R}^{N_{z}}$ and $\lambda(x, u) \in \mathbb{R}^{N_{u} \times N_{\theta}}$ are locally Lipschitz functions. It is assumed $(5 \mathrm{~b})$ is an explicit equation of $z$, and that $x$ is measured.

The system (2) can be written on the form (5) using

$$
\begin{aligned}
& x=\left[\begin{array}{lll}
p_{p}, & p_{c}, & q_{\mathrm{bit}}
\end{array}\right]^{\top}, u=\left[\begin{array}{lll}
q_{p}, & q_{\mathrm{bpp}}, & u_{c}
\end{array}\right]^{\top},
\end{aligned}
$$

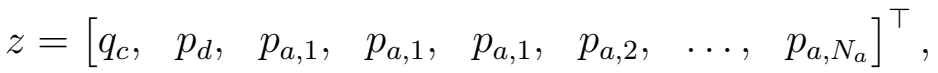

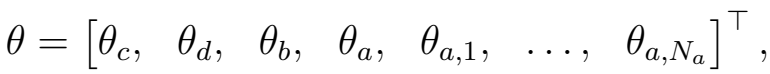

$$
\begin{aligned}
& \alpha(x, u)=\left[\begin{array}{c}
\frac{\beta_{d}}{V_{d}}\left(x_{3}-u_{1}\right) \\
\frac{\beta_{a}}{V_{a}}\left(x_{3}+u_{2}\right) \\
\frac{1}{M}\left(x_{1}-x_{2}-\left(\rho_{a}-\rho_{d}\right) g h_{\mathrm{TVD}}\right)
\end{array}\right],
\end{aligned}
$$




$$
\begin{aligned}
& \beta(x, u)=\left[\begin{array}{cccccc}
0 & 0 & 0 & 0 & & 0 \\
-\frac{\beta_{a}}{V_{a}} q_{c}\left(x_{1}, u_{3}\right) & 0 & 0 & 0 & \cdots & 0 \\
-\frac{1}{M} f_{d}\left(x_{3}\right) & -\frac{1}{M} f_{b}\left(x_{3}\right) & -\frac{1}{M} f_{a}\left(x_{3}\right) & 0 & & 0
\end{array}\right] \\
& \eta(x, z)=\left[0, x_{1}+G_{d}, z_{2}, x_{2}+G_{a}, z_{6}+G_{a, 1}, \ldots, x_{2}+G_{a, N_{a}}\right]^{\top}, \\
& \lambda(x, u)=\operatorname{diag}\left\{q_{c}\left(x_{1}, u_{3}\right),-f_{d}\left(x_{3}\right),-f_{b}\left(x_{3}\right), f_{a}\left(x_{3}\right), \ldots, f_{a}\left(x_{3}\right)\right\} .
\end{aligned}
$$

Theorem 1 (Willersrud and Imsland [14]). Given an observer on the form

$$
\begin{aligned}
& \dot{\hat{x}}=\alpha(x, u)+\beta(x, u) \hat{\theta}-K_{x}(\hat{x}-x), \\
& \dot{\hat{\theta}}=-\Gamma \beta^{\top}(x, u)(\hat{x}-x)-\Lambda \lambda^{\top}(x, u)(\hat{z}-z), \\
& \hat{z}=\eta(x, z)+\lambda(x, u) \hat{\theta},
\end{aligned}
$$

where $K_{x}, \Lambda, \Gamma>0$ are tuning matrices, and with $\dot{\theta}=0$. Let $e_{x}=\hat{x}-x$ and $e_{\theta}=\hat{\theta}-\theta$ be variables for the error dynamics, where $e=\left[\begin{array}{ll}e_{x}^{\top}, & e_{\theta}^{\top}\end{array}\right]^{\top}=0$ is an equilibrium point. Then $e=0$ is globally exponentially stable if

$$
\Gamma^{-1} \Lambda \lambda^{\top}(\cdot) \lambda(\cdot)-\beta^{\top}(\cdot) K^{\top} K \beta(\cdot)>k I_{N_{\theta}}
$$

for some constant $k>0$, where $I_{N_{\theta}} \in \mathbb{R}^{N_{\theta} \times N_{\theta}}$ is the identity matrix.

See [14] for proof of Thm. 1. Requirement (8) with $\lambda(\cdot), \beta(\cdot)$ given in (6) will be met if there is flow through the system, i.e., for a non-zero and bounded $x_{3}=q_{\mathrm{bit}}$, and bounded $x_{1}=p_{p}$.

\section{Multivariate statistical change detection, fault isolation and fault localization}

Detecting changes to the different estimated parameters in $\Theta_{D}, \Theta_{I}$ is done using a generalized likelihood ratio test, described in this section. The diagnosis problem is a set of stepwise problems: First detect that there is a change from normal and isolate which incident is causing this change, then isolate the incident to a particular section of the drill string and estimate its magnitude. See also Fig. 4. 
Problem 1 (Incident detection). Given a sampled time sequence of vectors of estimated parameters $\Theta_{D}(k)$, with change from known condition $\Theta_{D, 0}(k)$ to unknown $\Theta_{D, 1}(k)$ defined as $\Delta \Theta_{D}(k):=\Theta_{D, 1}(k)-\Theta_{D, 0}(k)$. Define the index set $\mathbb{N}_{N}:=\{i \in \mathbb{N}: 1 \leq i \leq N\}$ and let $i_{D} \in \mathbb{N}_{N_{f}}$ be the possible fault indices. Let a fault signature matrix be D, a unit magnitude fault vector be $f_{i_{D}}=[0, \ldots, 0,1,0, \ldots, 0]^{\top}$, element $i_{D}$ of which is non-zero when fault $i_{D}$ is present, and $v(k)$ be an unknown magnitude of change. Further, let the random vector $w(k)$ have independent and identically distributed samples with probability density $f(w)$, then distinguish between two hypotheses

$$
\begin{aligned}
\mathcal{H}_{0}: & \Delta \Theta_{D}(k)=0+w(k), & & \text { no fault present }, \\
\mathcal{H}_{1}^{D}: & \Delta \Theta_{D}(k)=D f_{i_{D}} v(k)+w(k), & & \text { a fault is present } .
\end{aligned}
$$

Problem 2 (Isolate type of incident). Given $\mathcal{H}_{1}^{D}$ has been accepted, determine that a particular fault $i_{D}^{*}$ is present of the possible faults $i_{D} \in \mathbb{N}_{N_{f}}$, by determining the best fit of (9b) for the different fault types.

Problem 3 (Isolate position of incident). Given that $i_{D}^{*}$ has been isolated. Let $G\left(i_{D}^{*}\right)$ be a known matrix associated with the isolated fault type $i_{D}^{*}, \Delta \Theta_{I}(k)$ be a vector of change in estimated parameters, $j_{I} \in \mathbb{N}_{N_{g}}$ be the possible fault positions, $f_{j_{I}}$ be a fault position vector with element $j_{I}$ equal to 1 for a fault in position $j$ and 0 otherwise, and $v(k)$ be the unknown magnitude of the change.

(A): Determine if localization of a fault is possible by distingushing between two hypotheses

$$
\begin{array}{lll}
\mathcal{H}_{0}: & \Delta \Theta_{I}(k)=0+w(k), & \text { localization not possible } \\
\mathcal{H}_{1}^{I}: & \Delta \Theta_{I}(k)=G\left(i_{D}^{*}\right) f_{j_{I}} v(k)+w(k), & \text { localization possible }
\end{array}
$$

(B): If hypothesis $\mathcal{H}_{1}^{I}$ is accepted, determine the most likely position $j_{I}^{*}$ of the positions $j_{I} \in \mathbb{N}_{N_{g}}$ along the pipe that explains the estimates (10b).

\subsection{Generalized likelihood ratio test}

The GLRT decision function uses the likelihood ratio of the probability density function at the two hypotheses of $\mathcal{H}_{0}$ and $\mathcal{H}_{1}$, and can be written as

$$
g(k)=\max _{k-N+1 \leq j \leq k-\tilde{N}} \ln \frac{\prod_{i=j}^{k} f\left(\Theta(i) ; \mathcal{H}_{1}\right)}{\prod_{i=j}^{k} f\left(\Theta(i) ; \mathcal{H}_{0}\right)} .
$$


using a data window $N$ to reduce computational cost, and $0 \leq \tilde{N}<N[31$, 32]. Distinguishing between the two hypotheses is done by using a threshold $h$ of the decision function $g(k)$,

$$
\begin{aligned}
& \text { accept } \mathcal{H}_{0}: g(k) \leq h, \\
& \text { accept } \mathcal{H}_{1}: g(k)>h .
\end{aligned}
$$

\subsection{Probability distribution of estimated flow and friction parameters}

The estimated parameters $\hat{\theta}$ from the adaptive observer $(7)$ were found to be multivariate $t$-distributed in [15], after the estimated parameters were white-filtered. The $t$-distribution is a generalization of the Gaussian distribution, with larger probability tails. This means that there is a higher probability of outliers compared to a Gaussian distribution. The $p$-variate $t$-distribution with center $\mu$, correlation matrix $S$ and $\nu \in(0, \infty]$ degrees of freedom has the joint probability density function

$$
f(x ; \mu, S, \nu)=\frac{\Gamma((p+\nu) / 2)}{\Gamma(\nu / 2)(\pi \nu)^{p / 2}|S|^{1 / 2}}\left[1+\frac{1}{\nu}(x-\mu)^{\top} S^{-1}(x-\mu)\right]^{-\frac{p+\nu}{2}},
$$

where $\Gamma(z)$ is the Gamma function. The parameter $\mu$ is the mean of $x$ when $\nu>1[33]$.

\subsection{GLRT with multivariate t-distribution}

If the mean $\mu$ is changing from $\mu_{0}$ to $\mu_{1}$, whereas the statistical parameters $S$ and $\nu$ are constant, the GLRT decision function $g(k) \in \mathbb{R}$ for the $t$-distribution (13) of vector variable $\Theta(k)$ was found in [15] to be given by

$$
\begin{array}{r}
g(k)=\max _{k-N+1 \leq j \leq k-\tilde{N}} \frac{p+\nu}{2} \sum_{i=j}^{k}\left[-\ln \left(1+\frac{1}{\nu}\left(\Theta(i)-\hat{\mu}_{1}\right)^{\top} S^{-1}\left(\Theta(i)-\hat{\mu}_{1}\right)\right)\right. \\
\left.+\ln \left(1+\frac{1}{\nu}\left(\Theta(i)-\mu_{0}\right)^{\top} S^{-1}\left(\Theta(i)-\mu_{0}\right)\right)\right],
\end{array}
$$

with maximum likelihood estimate of the mean after change given by

$$
\hat{\mu}_{1}=\frac{1}{k-j+1} \sum_{i=j}^{k} \Theta(i) .
$$




\subsection{Isolate type of incident}

The problem of isolating type of incident defined in Problem 2 is to find $i_{D}^{*}$ of the possible fault types. Let $D$ be the fault signature matrix with unit column vectors $D_{i}$ defined by

$$
D_{i}:=\frac{K_{D} \Upsilon_{D, i}}{\left\|K_{D} \Upsilon_{D, i}\right\|},
$$

where the column vector $\Upsilon_{D, i}$ of $\Upsilon_{D}$ is the change direction of incident $i_{D}$, and $K_{D}$ are the relative change magnitudes used to scale changes to have approximately similar effects on magnitude $v$. Based on Tab. 1, the possible change directions $\Upsilon_{D}$ for $\Theta_{D}$ are

$$
\Upsilon_{D}=\left[\begin{array}{ccccc}
0 & -1 & 0 & 0 & 0 \\
0 & -1 & 0 & 1 & 0 \\
-1 & -1 & 1 & 0 & 1 \\
-1 & 0 & 1 & 0 & 0
\end{array}\right]
$$

corresponding to the fault types lost circulation $\left(i_{D}=1\right)$, drillstring washout $\left(i_{D}=2\right)$, gas influx $\left(i_{D}=3\right)$, bit plugging $\left(i_{D}=4\right)$, and pack-off $\left(i_{D}=5\right)$, respectively.

Determining correct magnitudes $K_{D}$ can be difficult without prior data of the incidents. Nevertheless, knowledge of certain range of values is maybe possible based on physical considerations. It is assumed that the relative change between friction parameters $\theta_{d}, \theta_{b}$ and $\theta_{d}$ is approximately equal. Furthermore, using $(2 \mathrm{k})$ with known friction and flow, relative change between the friction parameters $\theta_{d}, \theta_{b}, \theta_{d}$, and change of flow $\Delta q$ is approximately $1 / 1000$, giving the diagonal matrix of relative change

$$
K_{D}=\operatorname{diag}\{1,1,1,1 / 1000\} .
$$

The type of fault $i_{D}^{*}$ is isolated using a maximum least square solution of magnitude $v$ given by (9b) for each column vector $D_{i}$ in $D$, where mean $\mathrm{E}\left(\Delta \Theta_{D}\right)=\left(\hat{\mu}_{1}^{D}-\mu_{0}^{D}\right)$, giving

$$
i_{D}^{*}=\arg \max _{i} \frac{D_{i}^{\top}\left(\hat{\mu}_{1}^{D}-\mu_{0}^{D}\right)}{D_{i}^{\top} D_{i}} .
$$




\subsection{Isolating position of incident}

The position of the incident refers to the position between two pressure measurements in the annulus, where $j_{I}$ indicates an incident between pressure sensor $p_{a, j}$ and $p_{a, j+1}$. The different type of incidents $i_{D} \in \mathbb{N}_{N_{f}}$ will affect the estimated parameters $\Theta_{I}$ used for isolation differently. As stated in Problem 3 , it is thus necessary to first determine type of incident $i_{D}^{*}$ and then isolate the position $j_{I}^{*}$. Let $G\left(i_{D}\right)$ be the localization matrix associated with fault type $i_{D}$ with unit column vectors $G_{j}\left(i_{D}\right)$ defined as

$$
G_{j}\left(i_{D}\right):=\frac{K_{I} \Upsilon_{I, j}\left(i_{D}\right)}{\left\|K_{I} \Upsilon_{I, j}\left(i_{D}\right)\right\|}
$$

where $\Upsilon_{I, j}\left(i_{D}\right)$ is the $j$-th column vector of the localization change direction matrix $\Upsilon_{I}\left(i_{D}\right)$ associated with fault type $i_{D}$, and $K_{I}$ is a diagonal matrix of relative change magnitudes.

Similarly to (19), the position $j_{I}^{*}$ of the fault is isolated finding the maximum least square solution to (10b), giving

$$
j_{I}^{*}=\arg \max _{j} \frac{G_{j}^{\top}\left(i_{D}^{*}\right)\left(\hat{\mu}_{1}^{I}-\mu_{0}^{I}\right)}{G_{j}\left(i_{D}^{*}\right)^{\top} G_{j}\left(i_{D}^{*}\right)} .
$$

It is assumed that the magnitude of change of each estimated parameter is equal for a given incident, giving $K_{I}=I$, where $I$ is the identity matrix. For the case of lost circulation $\left(i_{D}=1\right)$, drillstring washout $\left(i_{D}=2\right)$, and pack-off $\left(i_{D}=5\right)$, the change direction matrices are, respectively, given by

$$
\begin{aligned}
& \Upsilon_{I}(1)=\left[\begin{array}{cccc}
-1 & 0 & 0 & 0 \\
-1 & -1 & 0 & 0 \\
-1 & -1 & -1 & 0 \\
-1 & -1 & -1 & -1
\end{array}\right], \Upsilon_{I}(2)=\left[\begin{array}{cccc}
1 & -1 & -1 & -1 \\
0 & 1 & -1 & -1 \\
0 & 0 & 1 & -1 \\
0 & 0 & 0 & 1
\end{array}\right] \\
& \Upsilon_{I}(5)=\left[\begin{array}{llll}
1 & 0 & 0 & 0 \\
0 & 1 & 0 & 0 \\
0 & 0 & 1 & 0 \\
0 & 0 & 0 & 1
\end{array}\right],
\end{aligned}
$$

where vectors are determined based on discussions in Sec. 5. Isolation of gas influx $\left(i_{D}=3\right)$ is not well suited for the current model, and is hence not included. Bit plugging $\left(i_{D}=4\right)$ does not need additional isolation. 


\subsection{Determining thresholds}

Thresholds can be found based on specified probability of false alarms $P_{\mathrm{FA}}$ [28], by determining the distribution of the GLRT test statistic $g(k)$ for data under $\mathcal{H}_{0}$, see, e.g., $[34,35]$. In [15] the GLRT test statistic (14) was found to have a good fit to the Weibull distribution. The Weibull distribution has the cumulative distribution function $F(x ; \alpha, \beta)$ and probability density function $f(x ; \alpha, \beta)$ given by

$$
\begin{array}{ll}
F(x ; \alpha, \beta)=1-e^{-(x / \alpha)^{\beta}}, & x \geq 0, \\
f(x ; \alpha, \beta)=\frac{\beta}{\alpha}\left(\frac{x}{\alpha}\right)^{\beta-1} e^{-(x / \alpha)^{\beta}}, & x \geq 0,
\end{array}
$$

where $\alpha>0$ is the scale and $\beta>0$ the shape parameter.

Let $P_{\mathrm{FA}}$ be the probability of false alarm under $\mathcal{H}_{0}$. Then the inverse cumulative distribution function gives a threshold $h$ with given probability $P_{\mathrm{FA}}$

$$
h=Q\left(1-P_{F A} ; \mathcal{H}_{0}, \alpha_{0}, \beta_{0}\right)=\beta_{0}\left(-\ln \left(P_{F A}\right)\right)^{1 / \alpha_{0}} .
$$

The probability of detecting a fault under the alternative hypothesis $\mathcal{H}_{1}$ with probability $P_{D}$ for a given threshold $h$ is

$$
P_{D}=1-F\left(h ; \mathcal{H}_{1}, \alpha_{1}, \beta_{1}\right)=e^{-\left(x / \alpha_{1}\right)^{\beta_{1}}} .
$$

Knowledge of data under $\mathcal{H}_{1}$ is needed to find $\alpha_{1}, \beta_{1}$, and thus $P_{D}$.

\section{Diagnosis of downhole incidents in flow loop data}

The suggested incident diagnosis method, illustrated in Fig. 4, is tested on data from five different cases from the test rig: drillstring washout; lost circulation; two cases of gas influx; and bit plugging. Data is sampled at 10 $\mathrm{Hz}$ and white-filtered using a third order filter. The computational burden of updating the observer (7) is well within real-time capability.

Estimation of pump and choke pressure, as well as pump and choke flow, is shown for all test cases in Fig. 7. Since pressures and choke flow are measured, these estimates closely follow the process as expected. Since measured bit flow is assumed equal to pump flow, estimated bit flow closely follows the pump flow. The estimated parameters in (2) are plotted in Fig. 8, which will determine $\Theta_{D}$ and $\Theta_{I}$ given by (4). Measurements indicating the time of 
the emulated incidents are plotted in Fig. 9. Valve position for bit plugging emulation was not measured and is not shown. This information is shown for reference only, the emulated incidents are not known to the diagnosis algorithm.

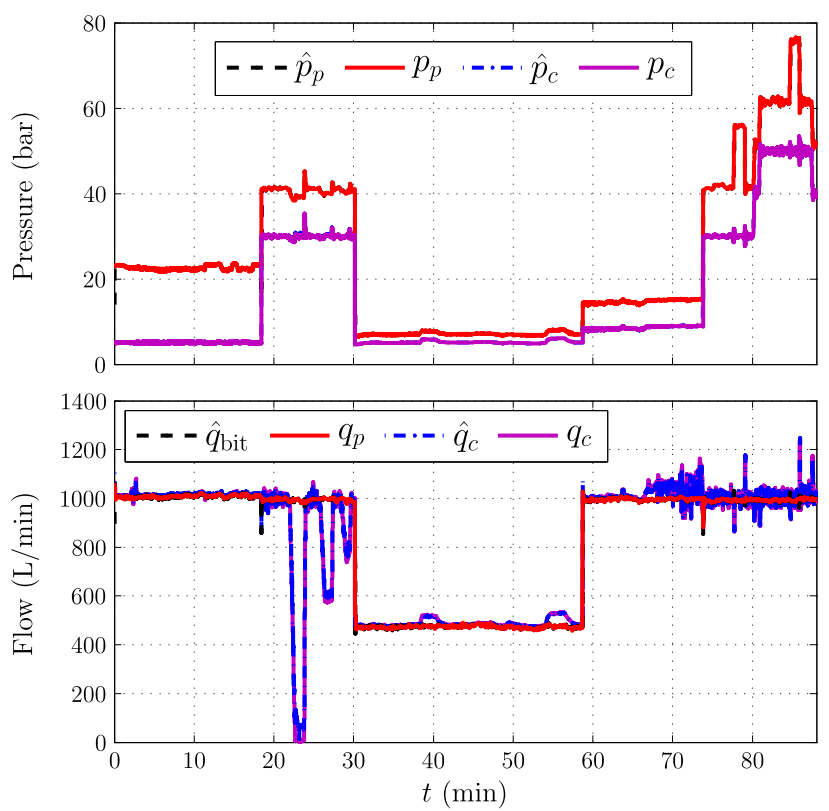

Figure 7: State estimation of pressure and flow during washout, loss, gas influx, and bit plugging.

The plots in Figs. 7, 8 and 9 show a concatenation of the five different data sets logged at the test rig. Logging was not continuously available and incidents were not always injected in chronological order. Although the different cases were run on the same experimental rig, physical conditions differ between the experiments and there are therefore differences in the state and parameter estimates between the individual cases. From Fig. 8 that shows parameter estimation, it is apparent that the distribution of test statistics under $\mathcal{H}_{0}$ differ from one experiment to another. In a real drilling process, the estimates would only have small variations during normal operation, and a $\mathcal{H}_{0}$ calibration could be made from data.

With differences between data sets, different values for the parameters in the $t$-distribution, i.e., $\mu_{0}, S$ and $\nu$, need be estimated. The $t$-distribution parameters under $\mathcal{H}_{0}$ are estimated using the ECME algorithm [36], using data from test conditions without any incidents. For all data sets $\nu>1$, 

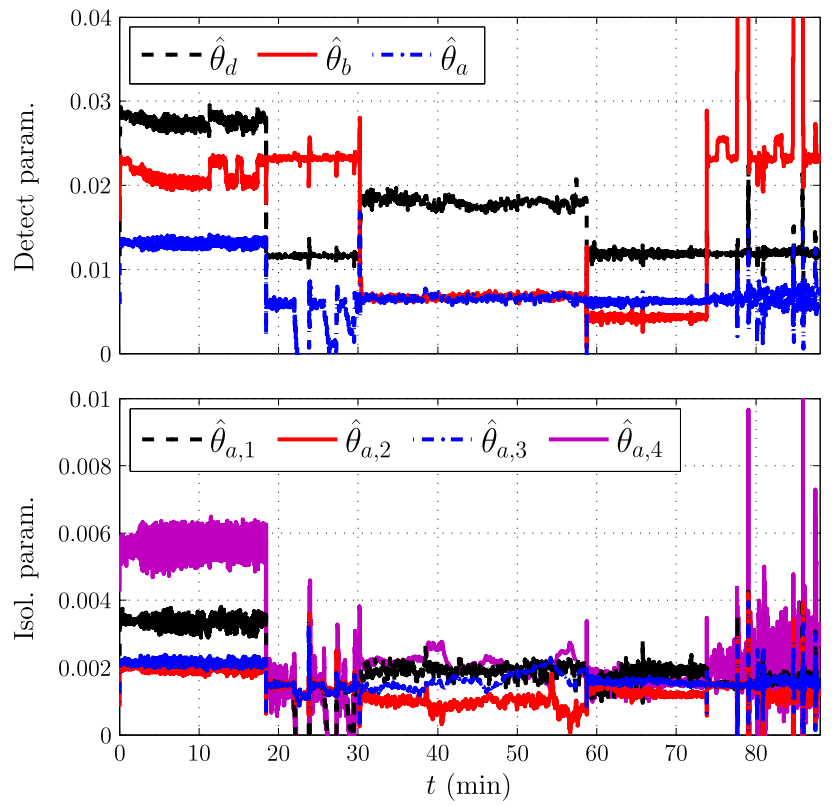

Figure 8: Parameter estimation during washout, loss, gas influx, and bit plugging.

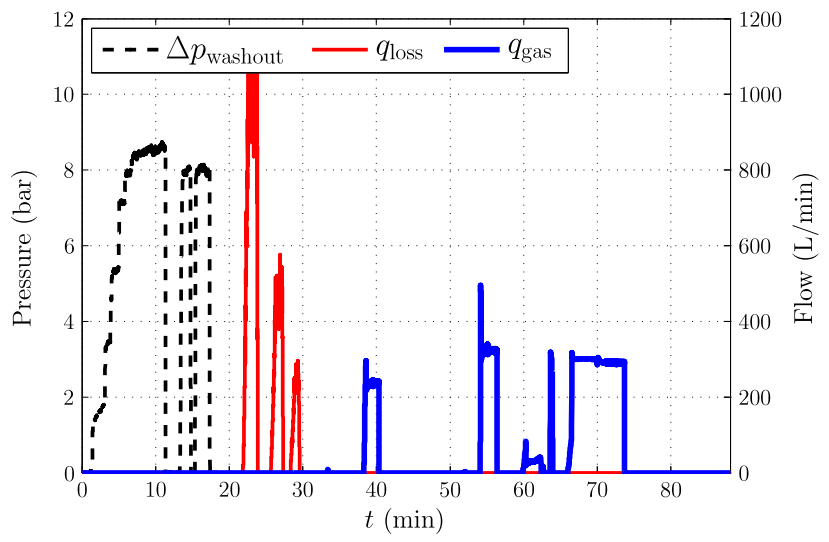

Figure 9: Actual incidents: drillstring washout, lost circulation, and gas influx. Bit plugging not measured. Information not available for diagnosis method. 
meaning that $\mu$ is the vector of mean values of parameters. Simultaneous adaptation and change detection could be used to track slowly varying process properties.

A related approach was presented in [37] where adaptation of model parameters was halted when a $\mathcal{H}_{1}$ condition was detected, and detection was based on a combination of change in parameters and change in the output estimation error between observer estimated output and measured output.

Threshold values listed in Tab. 2 are calculated using (24) with the following probabilities of false alarm

$$
P_{F A, D}=10^{-6}, \quad P_{F A, I}=10^{-4},
$$

during detection and type isolation, and localization, respectively.

The chosen GLRT window lengths given in number of samples are

$$
N_{D}=150, \quad N_{I}=400,
$$

where a shorter window for detection and type isolation is used to give a fast detection, while localization is based on $\Theta_{I}$ with less changes in the estimated parameters, necessitating a longer window. The necessity for a sufficiently long window size is shown in Fig. 10, showing $g\left(k ; \Theta_{D}\right)$ fitted to Weibull-distributions for the lost circulation case under $\mathcal{H}_{0}$ with no loss, and $\mathcal{H}_{1}$ with lost circulation of $300 \mathrm{~L} / \mathrm{min}$ shown at $29 \mathrm{~min}$ in Fig. 9. Two cases are plotted, namely the chosen window size of $N_{D}=150$ plotted with solid lines, and a shorter window $N_{D}=30$ plotted with dashed lines. Also plotted are the thresholds that give the same false alarm probability for the two window sizes. As Fig. 10 shows, in order to obtain high detection probability $\left(P_{D}\right)$ and a satisfactorily low false alarm rate $\left(P_{F}\right), \mathcal{H}_{0}$-data needs essentially to be below (to the left) of the threshold, and $\mathcal{H}_{1}$-data essentially to be above. This is the case for $N_{D}=150$, which is used in our analysis, but not for a five times shorter window, $N_{D}=30$. The window intervals we use in the analysis, see (27), are quite short $\left(N_{D}=150\right.$ is equivalent to a $15 \mathrm{~s}$ window and $N_{I}=400$ is equivalent to $40 \mathrm{~s}$ ), so the window size could easily be chosen much longer in a real drilling situation, albeit at the expense of slower detection.

This discussion illustrates the necessity of investigating the distribution of test statistics under both $\mathcal{H}_{0}$ and $\mathcal{H}_{1}$ in order to choose threshold and window size for a test, and the probability plot approach shown here provides a straightforward and easily applicable methodology. It is a prerequisite that 


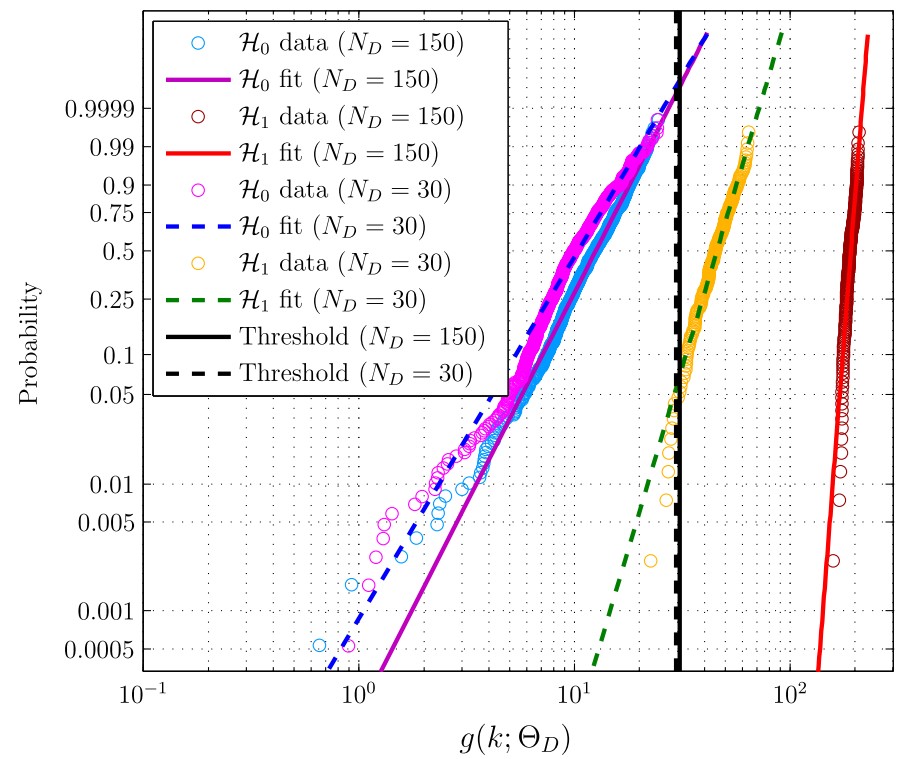

Figure 10: Weibull probability plot of decision function $g\left(k ; \Theta_{D}\right)$ under $\mathcal{H}_{0}$ and $\mathcal{H}_{1}$ with loss of $300 \mathrm{~L} / \mathrm{min}$ (at $29 \mathrm{~min}$ in Fig. 9), for window lengths $N_{D}=150$ (solid) and $N_{D}=30$ (dashed). Thresholds $h$ shown as vertical lines. $P_{F A}$ and $P_{D}$ are $1-$ Probability shown on the ordinate axis of the plot.

$\mathcal{H}_{0}$ and at least a few $\mathcal{H}_{1}$ data are available. If only $\mathcal{H}_{0}$ data are available, the minimum value of a fault that can be detected, with a given probability of detection, will be a function of threshold and window length.

With $P_{F A}$ and $N$ specified in (26) and (27), expected false alarm rates are 0.00024 per hour (2 per year) for detection and type isolation, and 0.009 per hour for localization. Since localization is made subject to prior detection, the localization false alarm does not have as high priority as that of detection. In addition, with a longer window size or a requirement that consecutive hypothesis evaluations confirm a detection, false alarm rates could be further reduced.

\subsection{Drillstring washout}

The first incident studied is a drillstring washout. This case was studied in [15], but extended in this paper to also include isolation of incident type. Detection and isolation is shown in Fig. 11 where the washout is correctly detected and isolated. The true position of the washout is at position 3, which is in the middle of the drillstring. See Fig. 3. The position is correctly 
Table 2: Threshold values for different cases.

\begin{tabular}{lcc}
\hline & $h_{\text {det }}$ & $h_{\text {isol }}$ \\
\hline Lost circulation $\left(f_{D, 1}\right)$ & 30.8 & 27.9 \\
Drillstring washout $\left(f_{D, 2}\right)$ & 64.0 & 82.4 \\
Gas influx A $\left(f_{D, 3}\right)$ & 56.3 & $\mathrm{~N} / \mathrm{A}$ \\
Gas influx B $\left(f_{D, 3}\right)$ & 48.4 & $\mathrm{~N} / \mathrm{A}$ \\
Bit plugging $\left(f_{D, 4}\right)$ & 57.6 & $\mathrm{~N} / \mathrm{A}$ \\
\hline
\end{tabular}

located after 2 minutes, seen in Fig. 12, with the alarm disappearing shortly in the beginning where leakage is fairly small. A multivariate Gaussian test on non-whitened estimates, see, e.g., $[27,20]$ is also shown in the figure.

Using the multivariate $t$-distribution, the probability of missed detection $P_{M}:=1-P_{D}$ for $g\left(k ; \Theta_{D}\right)$ between 2 and 3 minutes is $2.6 \times 10^{-4}$, using $P_{D}$ in $(25)$ and $P_{\mathrm{FA}}$ specified in (26). For localization with $g\left(k ; \Theta_{I}\right), P_{M}=0.106$. If the multivariate Gaussian distribution is used these values are $1.6 \times 10^{-3}$ and 0.80 , respectively, which is considerably higher. The $t$-distribution is hence providing better detection properties, where isolation in particular would be challenging using a multivariate Gaussian distribution in the GLRT decision function (11). This can also can be seen in Fig. 12, where $g\left(k ; \Theta_{I}\right)$ is lower using the Gaussian probability density function. The $g(k)$ value of the Gaussian distribution is scaled to have equal threshold $h$ as the $t$-distribution.

\subsection{Loss}

The next incident is loss of drilling fluid, happening just downstream the bit, which is position 1 in the annulus. The data contains three losses at different magnitudes of approximately $1000 \mathrm{~L} / \mathrm{m}, 500 \mathrm{~L} / \mathrm{m}$ and $300 \mathrm{~L} / \mathrm{min}$. See actual loss $q_{\text {loss }}$ plotted in Fig. 9. All of these losses are correctly detected and isolated, as seen in Fig. 13. Isolation of the position is also correctly found, as seen in Fig. 14. Also here the $t$-distribution gives better detection and isolation properties.

\subsection{Influx}

With gas in the system, some aspects of the model are no longer valid. In the model it is assumed incompressible single-phase flow with constant density in the annulus, whereas during a gas influx the flow will be two-phase and compressible with varying density. For such a case it is expected that 

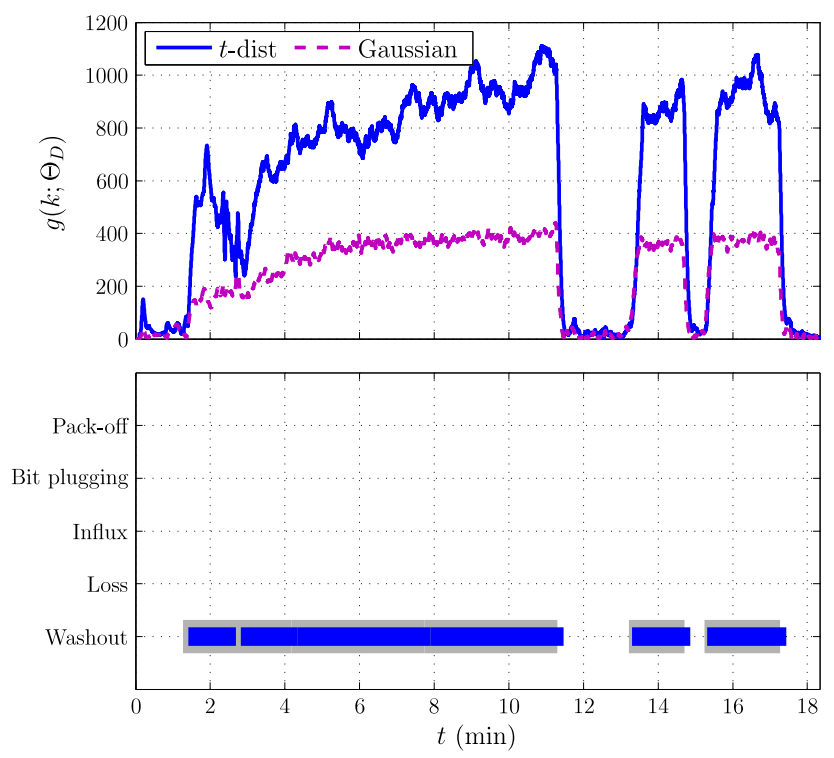

Figure 11: Detection and isolation of drillstring washout. GLRT plotted for $t$-distribution and Gaussian distribution. Gaussian GLRT scaled to have same threshold $h$ as the $t$ distribution. Actual incident shown in gray.
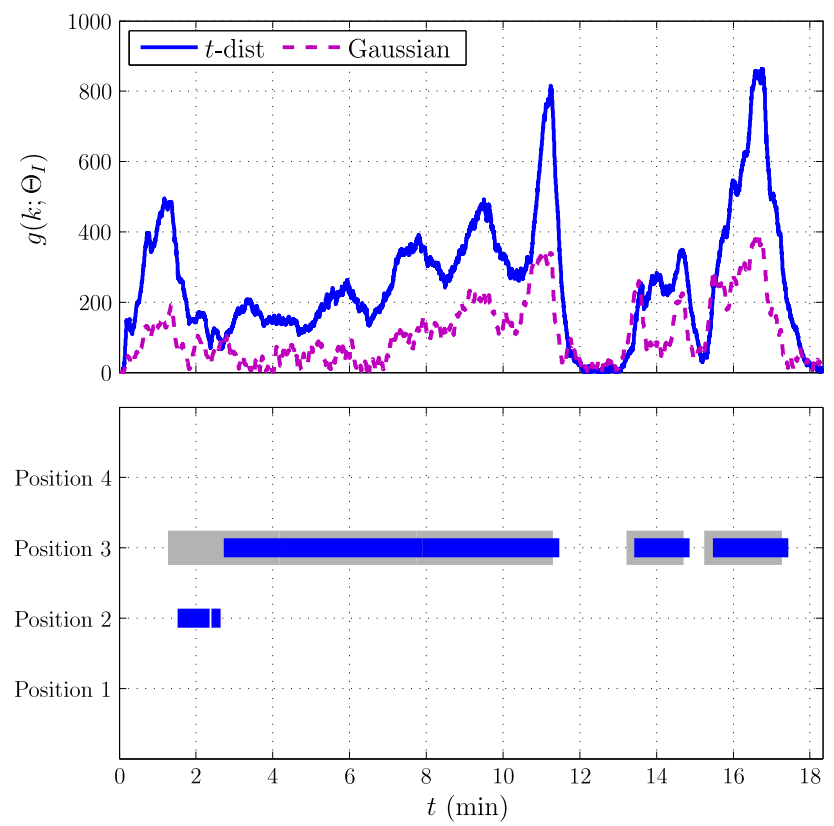

Figure 12: Localization of drillstring washout position. GLRT plotted for $t$ and Gaussian distribution (scaled). Actual position shown in gray. 

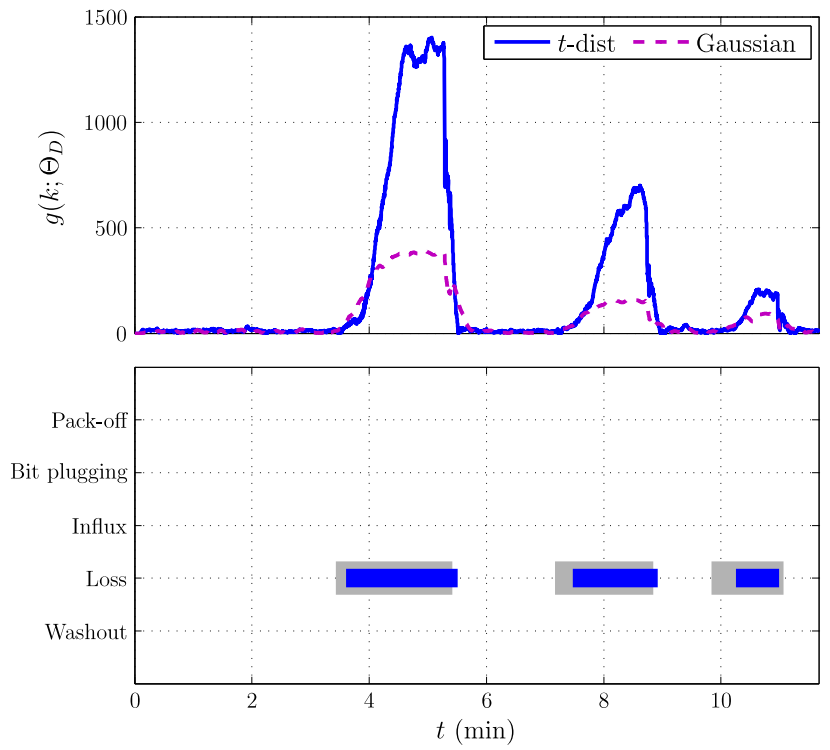

Figure 13: Detection and isolation of lost circulation. GLRT plotted for $t$ and Gaussian distribution (scaled). Actual incident shown in gray.
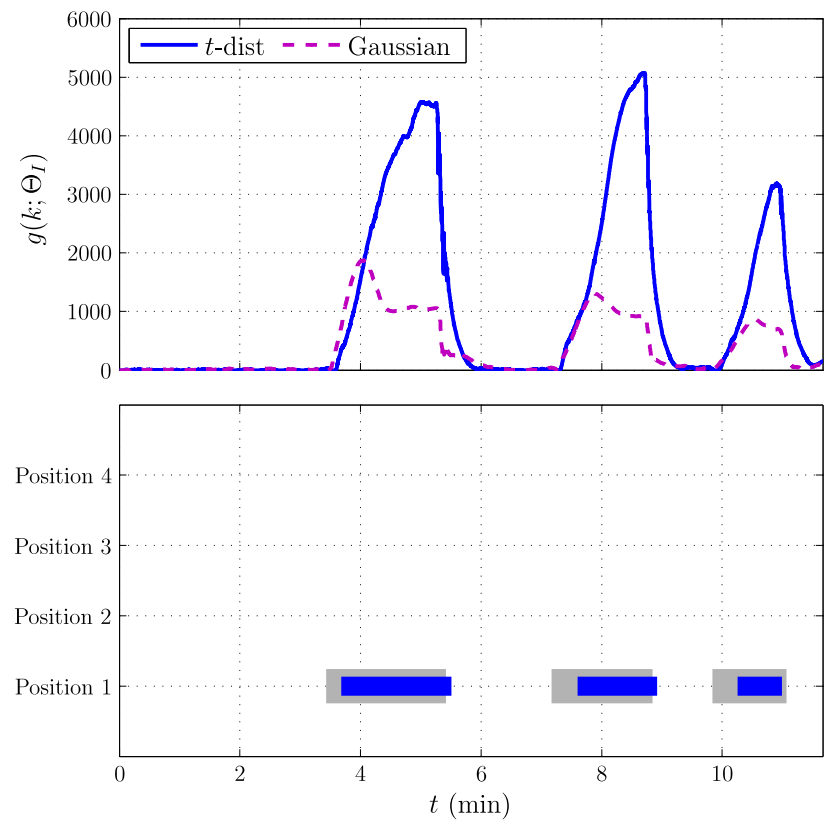

Figure 14: Localization of lost circulation position. GLRT plotted for $t$ and Gaussian distribution (scaled). Actual position shown in gray. 
a fit-for-purpose multi-phase model will describe the fluid dynamics better. Nevertheless, detection in the current framework is still tested, since it is important to have a diagnosis framework that correctly isolates the type of incident.

Detection and isolation of a gas influx is successfully detected in influx case A, shown in Fig. 15. Afterwards, there is gas present in the system which is not modeled, and thus causing a slightly increasing value of $g\left(k ; \Theta_{D}\right)$ after the first influx. However, this change is less that the threshold $h$ given in Tab. 2. Diagnosis in influx case B is shown in Fig. 16, which has three instances of gas influx, one small, one large over a small time period, and one large continuous influx, see Fig. 9 between 60 and 75 minutes. The small influx is successfully detected and isolated, although not at all time instances. The larger ones are also correctly diagnosed. After the first large influx, around the 5 minutes time stamp in Fig. 16, there are some false alarms of pack-off and washout. Since the actual incidents plotted is injection of gas, there is still gas in the system after injection. Transportation of gas is not modeled, and will affect friction and hydrostatic pressure as discussed in Sec. 5.3. Note that when a gas influx is detected in a real drilling operation, the well is typically shut in and normal drilling is stopped in order to remove the influx.

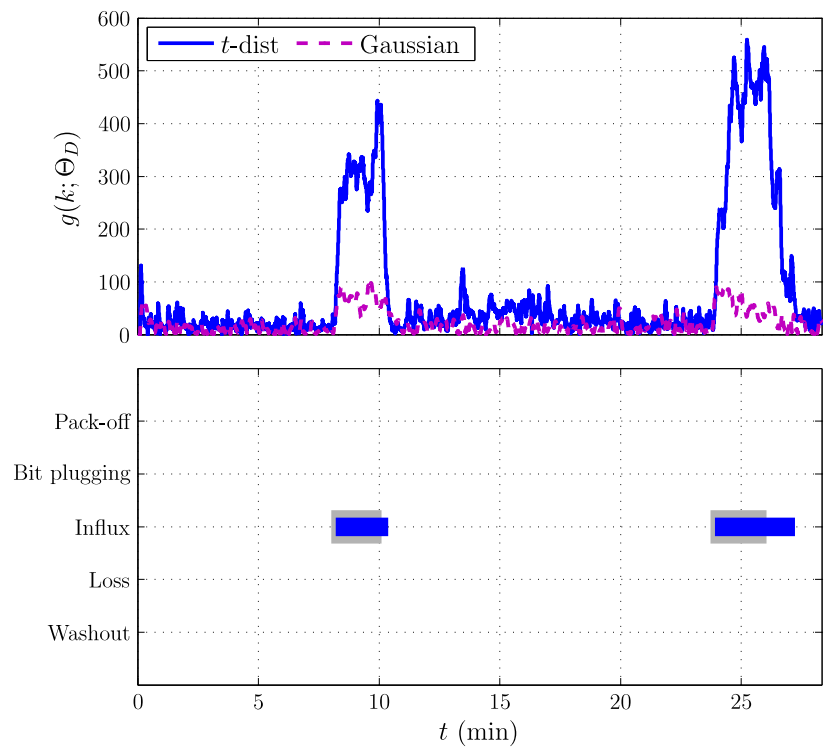

Figure 15: Detection and isolation of gas influx case A. GLRT plotted for $t$ and Gaussian distribution (scaled). Actual incident shown in gray. 

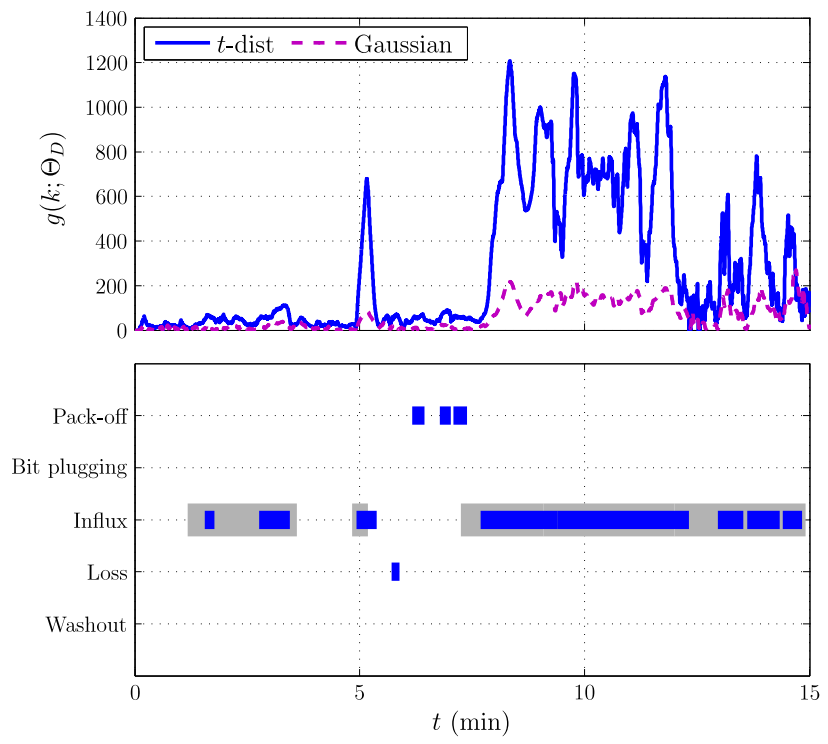

Figure 16: Detection and isolation of gas influx case B. GLRT plotted for $t$ and Gaussian distribution (scaled). Actual incident shown in gray.

\subsection{Bit plugging}

The last case studied is a plugging of the drill bit. In this case only changes in the estimated bit parameter $\hat{\theta}_{b}$ is expected. By using a multivariate method, changes to all signals in $\Theta_{D}$ can be tested, determining that in fact the bit parameter is the only one changing. Detection and isolation is shown in 17 , where all pluggings are detected, with two large pluggings and two small. Studying $\hat{\theta}_{b}$ from 75 to 88 minutes in Fig. 8, the major pluggings will be possible to detect without a statistical method, whereas the smaller ones may be difficult to separate from noise and process disturbances. However, as argued, changes to all parameters should be considered in order to correctly isolate the type of incident, which is best solved with a multivariate statistical method.

\subsection{Estimation of incident magnitude}

In addition to indicating that an incident is present and finding out where it is, it can be valuable for the operator to get information about the magnitude of the incident. Especially important to know is the magnitude of loss or influx. Estimation of fluid loss to the formation is shown in Fig. 18, which for an incompressible fluid is the difference in flow in and out of the 

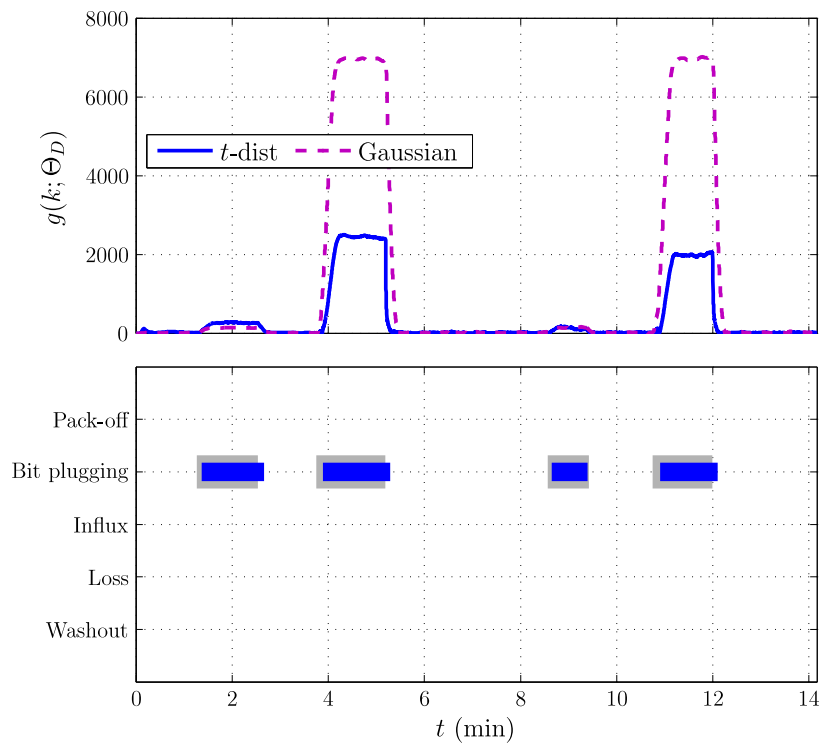

Figure 17: Detection and isolation of bit plugging. GLRT plotted for $t$ and Gaussian distribution (scaled). Actual incident shown in gray.

well. This plot shows that the loss magnitude is correctly estimated, and together with detection and isolation, information about the loss is well diagnosed. Gas influx is more challenging for the current model, since gas is not modeled. Drillstring washout and bit plugging magnitude is not directly measured, and cannot be verified. Nevertheless, bit plugging magnitude is possible to estimate based on changes to $\hat{\theta}_{b}$. Magnitude of drillstring washout flow was calculated in [15], although the value of washout flow could not be verified from data due to lack of washout flow measurement.

\section{Discussion}

The need for statistical change detection was evident from the parameter estimates shown in Fig. 8, where most of the incidents would be rather difficult to detect directly from the estimates. An exception is two of the four bit pluggings, where the estimated bit parameter $\hat{\theta}_{b}$ has a large change. However, to ensure that the incident was indeed a bit plugging, the whole process should be considered, i.e., a multivariate detection algorithm was needed.

Comparing drillstring washout and lost circulation in the schematic overview in Fig. 6, and in Tab. 1, shows that the two incidents have equal effect on the 


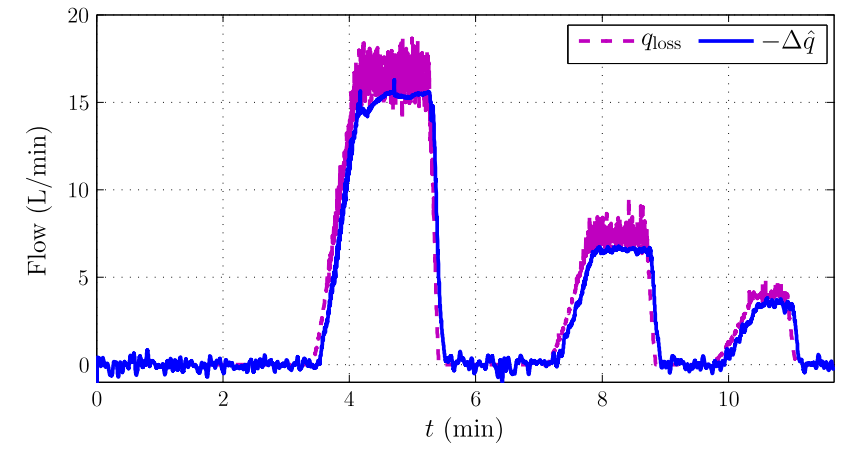

Figure 18: Estimation $(-\Delta \hat{q})$ and measurement $\left(q_{\text {loss }}\right)$ of lost circulation. Measurement not known to estimation algorithm.

annulus parameter $\hat{\theta}_{a}$. The same applies for gas influx if the pressure drop is gravity dominated. Separation of the incidents require one to consider changes to all signals in $\Theta_{D}$.

The proposed methodology successfully detects and isolates the different cases of drillstring washout, lost circulation, gas influx, and bit plugging. This represents a significant improvement over the results reported in [16], where isolation was uncertain. Reasons were that in [16], change to each parameter was considered separately, and that a Gaussian-based detector was used on the non-whitened estimates.

Isolation of the position is based on changes to estimated friction in the annulus, using distributed pressure measurements. With an increased number of measurements, the distance between them decreases, and frictional pressure drop decreases. It is therefore even more difficult to detect changes, making a statistical change detection algorithm necessary if changes to estimated parameters should be detected. For the drillstring washout case, the position is correctly isolated, except for a few minutes in the beginning with low washout rates. In the loss case, the position is correctly isolated for all three losses, also for the smaller loss. Isolation for the gas influx case was not considered. As discussed in Sec. 5.3, changes to pressure drop with a multi-phase flow is dependent on well geometry, where the friction drop could either be gravity dominated or friction dominated. Changes in $\Theta_{I}$ is thus dependent on the geometry, and where in the well the gas is located. This motivates the need for a fit-for-purpose model in order to isolate the position of the gas influx. Nevertheless, the main concern in drilling with respect to gas influx is to detect that it is happening, which the method 
successfully does.

It is shown in [15] that the white-filtered estimates are $t$-distributed. Using the dedicated $t$-distribution change detector gave superior results over the Gaussian detector for all cases except the bit plugging. This superiority is especially important for the isolation, where small changes in parameters were experienced.

\section{Conclusion}

Fault diagnosis of downhole incidents during oil and gas drilling was successfully done in this paper by estimating friction parameters and flow rates. Changes to estimates were detected by a multivariate generalized likelihood ratio test, considering the entire set of estimated well parameters simultaneously. Isolation of incident type and position was achieved by determining the direction of change of the estimated parameters. Data from a medium-scale horizontal flow loop of $1400 \mathrm{~m}$ was used to test the fault diagnosis method. Parameter and state estimates from data were found to have a non-Gaussian, $t$-distributed noise component, and this was utilized in the dedicated multivariate statistical change detection algorithm, developed specifically for this

distribution. Thresholds were determined based on specified probabilities of false alarms. Diagnosis of drillstring washout, lost circulation, gas influx, and bit plugging were tested. All of these cases were successfully detected and isolated during the occurrence of the incident. A multi-phase flow model should be considered if isolation of gas influx position is required, whereas the position was correctly isolated for drillstring washout, fluid loss, and bit plugging.

\section{Acknowledgment}

Financial support from Statoil ASA and the Norwegian Research Council (NFR project 210432/E30 Intelligent Drilling) is gratefully acknowledged. Experimental data are results from ongoing internal research in the department Intelligent Drilling in Statoil RDI. The authors acknowledge the work done by Henrik Manum, Glenn-Ole Kaasa, Jon Myklebost and Qin Li in planning and conducting experiments. 


\section{References}

[1] J.-M. Godhavn, Control Requirements for Automatic Managed Pressure Drilling System, SPE Drilling and Completion 25 (3) (2010) 336-345.

[2] D. Hargreaves, S. Jardine, B. Jeffryes, Early Kick Detection for Deepwater Drilling: New Probabilistic Methods Applied in the Field, in: Proc. SPE Annu. Tech. Conf. and Exhib., SPE 71369, New Orleans, LA, 2001.

[3] H. Santos, E. Catak, J. Kinder, P. Sonnemann, Kick Detection and Control in Oil-Based Mud: Real Well-Test Results Using Microflux Control Equipment, in: Proc. SPE/IADC Drilling Conf., SPE 105454, Amsterdam, The Netherlands, 2007.

[4] J. Gravdal, M. Nikolaou, Ø. Breyholtz, L. Carlsen, Improved Kick Management During MPD by Real-Time Pore-Pressure Estimation, SPE Drilling and Completion 25 (4) (2010) 4-7.

[5] E. Hauge, O. M. Aamo, J.-M. Godhavn, G. Nygaard, A novel modelbased scheme for kick and loss mitigation during drilling, J. Process Control 23 (4) (2013) 463-472.

[6] A. T. Bourgoyne Jr., M. E. Chenevert, K. K. Millheim, F. Young, Applied Drilling Engineering, 2nd Edition, SPE, 1986.

[7] C. Dalton, M. Paulk, G. Stevenson, The Benefits of Real-Time Downhole Pressure and Tension Data With Wired Composite Tubing, J. Canadian Petroleum Technology 42 (5).

[8] K. Macdonald, J. Bjune, Failure analysis of drillstrings, Eng. Failure Analysis 14 (8) (2007) 1641-1666.

[9] E. Cayeux, B. Daireaux, E. Dvergsnes, A. Leulseged, B. Bruun, M. Herbert, Advanced Drilling Simulation Environment for Testing New Drilling Automation Techniques and Practices, SPE Drilling and Completion 27 (4) (2012) 6-8.

[10] E. Cayeux, E. W. Dvergsnes, G. Sælevik, Early Symptom Detection on the Basis of Real-Time Evaluation of Downhole Conditions : Principles and Results From Several North Sea Drilling Operations, SPE Drilling and Completion 27 (4) (2012) 546-558. 
[11] P. Skalle, A. Aamodt, O. E. Gundersen, Detection of Symptoms for Revealing Causes Leading to Drilling Failures, SPE Drilling and Completion 28 (2) (2013) 182-193.

[12] T. O. Gulsrud, R. Nybø, K. S. Bjørkevoll, Statistical Method for Detection of Poor Hole Cleaning and Stuck Pipe, in: Proc. of Offshore Europe, SPE 123374, Aberdeen, UK, 2009.

[13] A. M. Nagy-Kiss, G. Schutz, Estimation and diagnosis using multimodels with application to a wastewater treatment plant, J. Process Control 23 (10) (2013) 1528-1544.

[14] A. Willersrud, L. Imsland, Fault Diagnosis in Managed Pressure Drilling Using Nonlinear Adaptive Observers, in: Proc. European Control Conference, Zürich, Switzerland, 2013, pp. 1946-1951.

[15] A. Willersrud, M. Blanke, L. Imsland, A. Pavlov, Drillstring Washout Diagnosis using Friction Estimation and Statistical Change Detection, IEEE Trans. Control Syst. Technol. (conditionally accepted).

[16] A. Willersrud, L. Imsland, A. Pavlov, G.-O. Kaasa, A Framework for Fault Diagnosis in Managed Pressure Drilling Applied to Flow-Loop Data, in: Dynamics and Control of Process Systems (DYCOPS), Mumbai, India, 2013, pp. 625-630.

[17] D. Veeningen, J. Palmer, G. Steinicke, J. Saenz, T. Hansen, From Field Test to Successful Integration of Broadband Drillstring System for Offshore Extended Reach Wells, in: Proc. SPE Annu. Tech. Conf. and Exhib., SPE 151386, San Diego, CA, 2012.

[18] R. Isermann, P. Ballé, Trends in the application of model-based fault detection and diagnosis of technical processes, Control Engineering Practice 5 (5) (1997) 709-719.

[19] J. Chen, R. J. Patton, Robust Model-Based Fault Diagnosis for Dynamic Systems, Springer, Boston, MA, 1999.

[20] M. Blanke, M. Kinnaert, J. Lunze, M. Staroswiecki, Diagnosis and faulttolerant control, 2nd Edition, Springer, Berlin, 2006. 
[21] S. X. Ding, Model-based Fault Diagnosis Techniques, Springer, Berlin, 2008.

[22] S. Yoon, J. F. MacGregor, Fault diagnosis with multivariate statistical models part I: using steady state fault signatures, J. Process Control 11 (4) (2001) 387-400.

[23] S. Yin, S. X. Ding, A. Haghani, H. Hao, P. Zhang, A comparison study of basic data-driven fault diagnosis and process monitoring methods on the benchmark Tennessee Eastman process, J. Process Control 22 (9) (2012) 1567-1581.

[24] S. X. Ding, Data-driven design of monitoring and diagnosis systems for dynamic processes: A review of subspace technique based schemes and some recent results, J. Process Control 24 (2) (2014) 431-449.

[25] J. Yu, A particle filter driven dynamic Gaussian mixture model approach for complex process monitoring and fault diagnosis, J. Process Control 22 (4) (2012) 778-788.

[26] P. Frank, X. Ding, Survey of robust residual generation and evaluation methods in observer-based fault detection systems, J. Process Control 7 (6) (1997) 403-424.

[27] M. Basseville, I. V. Nikiforov, Detection of Abrupt Changes: Theory and Applications, Prentice Hall, Englewood Cliffs, NJ, 1993.

[28] S. M. Kay, Fundamentals of Statistical Signal Processing: Detection Theory, Prentice Hall, Upper Saddle River, NJ, 1998.

[29] G.-O. Kaasa, Ø. N. Stamnes, O. M. Aamo, L. Imsland, Simplified Hydraulics Model Used for Intelligent Estimation of Downhole Pressure for a Managed-Pressure-Drilling Control System, SPE Drilling and Completion 27 (1) (2012) 127-138.

[30] A. R. Hasan, C. S. Kabir, A Study of Multiphase Flow Behavior in Vertical Wells, SPE Production Engineering 3 (2) (1988) 263-272.

[31] A. Willsky, H. Jones, A generalized likelihood ratio approach to the detection and estimation of jumps in linear systems, IEEE Trans. Autom. Control 21 (1) (1976) 108-112. 
[32] T. L. Lai, Sequential Changepoint Detection in Quality Control and Dynamical Systems, J. Roy. Statistical Soc. 57 (4) (1995) 613-658.

[33] S. Kotz, S. Nadarajah, Multivariate t-distribution and their applications, Cambridge University Press, Cambridge, United Kingdom, 2004.

[34] R. Galeazzi, M. Blanke, N. K. Poulsen, Early Detection of Parametric Roll Resonance on Container Ships, IEEE Trans. Control Syst. Technol. 21 (2) (2013) 489-503.

[35] S. Hansen, M. Blanke, Diagnosis of Airspeed Measurement Faults for Unmanned Aerial Vehicles, IEEE Trans. Aerosp. Electron. Syst. 50 (1).

[36] C. Liu, D. B. Rubin, ML estimation of the t distribution using EM and its extensions, ECM and ECME, Statistica Sinica 5 (1995) 19-39.

[37] M. Blanke, S. Hansen, Towards self-tuning residual generators for UAV control surface fault diagnosis, in: Proc. Conf. Control Fault-Tolerant Systems, Nice, France, 2013, pp. 37-42. 